\title{
Review
}

\section{Application of Non-Thermal Plasma to Fungal Resources}

\author{
Mayura Veerana ${ }^{1,+}+$, Nannan Yu ${ }^{1,+}$, Wirinthip Ketya ${ }^{1,+}$ and Gyungsoon Park ${ }^{1,2, *}$ \\ 1 Plasma Bioscience Research Center, Department of Plasma-Bio Display, Kwangwoon University, \\ Seoul 01897, Korea; mayuraveerana@gmail.com (M.V.); nannan19950326@163.com (N.Y.); \\ pam7794p@gmail.com (W.K.) \\ 2 Department of Electrical and Biological Physics, Kwangwoon University, Seoul 01897, Korea \\ * Correspondence: gyungp@kw.ac.kr; Tel.: +82-2-940-8324 \\ + These authors contributed equally to this work.
}

\begin{abstract}
In addition to being key pathogens in plants, animals, and humans, fungi are also valuable resources in agriculture, food, medicine, industry, and the environment. The elimination of pathogenic fungi and the functional enhancement of beneficial fungi have been the major topics investigated by researchers. Non-thermal plasma (NTP) is a potential tool to inactivate pathogenic and foodspoiling fungi and functionally enhance beneficial fungi. In this review, we summarize and discuss research performed over the last decade on the use of NTP to treat both harmful and beneficial yeast- and filamentous-type fungi. NTP can efficiently inactivate fungal spores and eliminate fungal contaminants from seeds, fresh agricultural produce, food, and human skin. Studies have also demonstrated that NTP can improve the production of valuable enzymes and metabolites in fungi. Further studies are still needed to establish NTP as a method that can be used as an alternative to the conventional methods of fungal inactivation and activation.
\end{abstract}

Keywords: non-thermal plasma; fungi; inactivation; activation; reactive species

check for

updates

Citation: Veerana, M.; Yu, N.; Ketya, W.; Park, G. Application of Non-Thermal Plasma to Fungal Resources. J. Fungi 2022, 8, 102. https://doi.org/10.3390/jof8020102

Academic Editor: David S. Perlin

Received: 21 December 2021

Accepted: 20 January 2022

Published: 21 January 2022

Publisher's Note: MDPI stays neutral with regard to jurisdictional claims in published maps and institutional affiliations.

Copyright: (c) 2022 by the authors. Licensee MDPI, Basel, Switzerland. This article is an open access article distributed under the terms and conditions of the Creative Commons Attribution (CC BY) license (https:// creativecommons.org/licenses/by/ $4.0 /)$.

\section{Introduction}

Fungi are the second-most abundant group of organisms after insects [1] and they play a significant role in agriculture, biomedicine, global health, and industry [2]. The number of fungal species on earth is estimated to be 11.7-13.2 million [3]. Over the last 100 years, the number of pathogenic fungi infecting plants, animals, and humans has increased [4]. Fungal pathogens cause some of the most lethal infectious diseases in humans and animals and fungal infections are responsible for the death of approximately 1.6 million people annually [5]. In the United States, fungal diseases were reported to cause economic losses of more than 7.2 billion dollars in 2017 [6]. The worldwide increase in invasive fungal infections, along with the spread of resistant fungal pathogens, is a serious threat to human health [6]. Fungi also produce toxins that are carcinogenic or are responsible for the decay or contamination of food products. On a global scale, fungal infections are jeopardizing food security by reducing crop yields or by resulting in the death of plants [7].

Despite the fact that some fungi are harmful, many fungi are used in industries, including the food and feed, pharmaceutical, paper and pulp, textile, detergent, and biofuel industries [2]. For centuries, humans have used fungi to ferment their foods. Yeasts are a central part of traditional and modern food manufacturing processes, wherein they are used to degrade waste and synthesize industrially useful products [8]. The benefits of fungal resources can be attributed to the enzymes produced by fungi. Fungal enzymes are associated with advantages, such as catalysis, rapid production and high yield, ease of genetic manipulation, and biodegradability [9]. Enzymes of fungal origin account for almost half of all commercial enzymes [9]. The market for filamentous fungi that produce plant-biomass-degrading enzymes is worth $€ 4.7$ billion and is predicted to double in the next ten years [10]. Biological enzymes (or biocatalysts), particularly those derived from microorganisms, have become essential for the rapidly growing biotechnology industry. 
As fungi exert both beneficial and harmful effects, it would help to functionally enhance beneficial fungi, while inactivating harmful fungi. Several technologies have been developed and tested to control pathogenic and spoiling fungi and improve the functional aspects of beneficial fungi $[11,12]$. During the last decade, non-thermal plasma (NTP) has shown great potential as a tool for inactivating pathogenic fungi [13-17]. Recent studies have also shown that NTP can improve the production of valuable fungal constituents, such as enzymes, by beneficial fungi [18-20]. In this review, we compile and describe use of NTP to control the growth of harmful fungi, while functionally enhancing beneficial fungi by focusing on research published since 2010.

\section{NTP Technology}

NTP is an ionized gas - the fourth state of matter-that generates reactive chemical species, such as reactive oxygen and nitrogen species, electrons, atoms, neutral molecules, charged species, and ultraviolet radiation [21]. NTP can be artificially generated from ambient air or certain gases at atmospheric and low pressures in the presence of a high voltage $(\sim \mathrm{kV})$ electric current. Radio frequency $(\mathrm{RF})$ power, Microwave (MW) power, alternating current (AC), or direct current (DC) can be used for plasma discharge [22]. Several configurations of plasma devices have been developed: dielectric barrier discharge (DBD) plasma, plasma jet, corona discharge plasma, and gliding arc discharge plasma [22]. Dielectric barrier discharge (DBD) plasma can be generated between two electrodes separated by a dielectric barrier after a high-voltage DC or AC current at high frequency $(\sim \mathrm{kHz})$ is applied. In a plasma jet, when working gas passes through two cylinder-type electrodes in which one electrode is connected to an electric power source at high frequency, it is ionized and exits through a nozzle with a jet-like appearance. Corona discharge plasma is generated between two or more needle-type or wire-type electrodes after high voltage is applied. Gliding arc discharge plasma is produced under high voltages at the spot where two electrodes are within a few millimeters of each other.

The non-thermal nature of NTP has enabled its biological applications [22,23]. NTP has been used in medicine (cancer therapy and wound healing), food industry (microbial decontamination of food), and agriculture (plant disinfection, enhancement of seed germination, and plant growth). The impact of the plasma treatment can be varied by modulating the voltage, treatment time, and gases used for plasma generation [22]. Various strategies are used to subject samples to plasma treatment. These include direct contact with a plasma jet, indirect contact with plasma-generated gas, and indirect contact with plasma-treated water (Figure 1).

The type of sample and the purpose of treatment determine the plasma device and treatment settings used. NTP has demonstrated dual effects (activation and inactivation) on cells, tissues, and organisms depending on the applied dose and species [22]. Plasma generates various levels of reactive oxygen species (ROS) and reactive nitrogen species (RNS) that activate or inactivate cells and tissues (as determined by the dose used) [24]. In medicine, NTP has been used to inactivate microorganisms and cancer cells and to activate cell proliferation and wound healing [25,26]. In agriculture, NTP has been used for disinfecting seeds and fresh produce as well as for enhancing seed germination and plant growth [22]. The dual effects of plasma indicate that it can serve as a promising tool for solving problems in medicine and agriculture. 


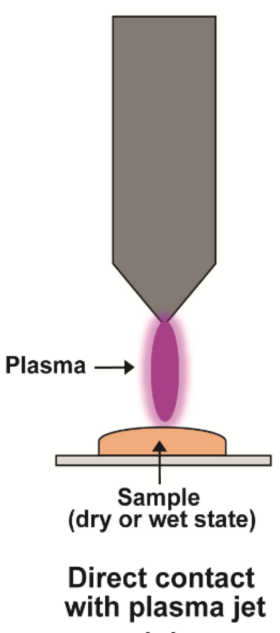

(a)

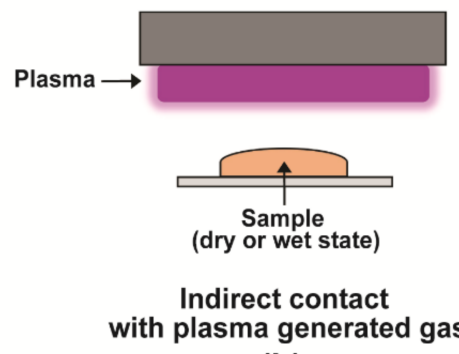

(b)

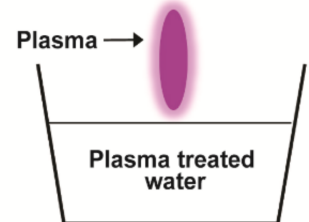

$\sqrt{2}$

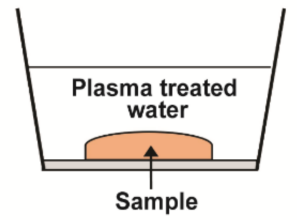

Indirect contact

with plasma treated water

(c)

Figure 1. Various strategies used for plasma treatment. Samples can be treated in a dry or wet state. (a) The sample is directly exposed to a plasma jet. (b) The sample is indirectly exposed to a gas produced from plasma. (c) The sample is submerged in plasma-treated water.

\section{Inactivation of Fungi Using NTP}

\subsection{Inactivation of Fungal Spores, Cells, and Biofilms In Vitro}

The antimicrobial activities of non-thermal atmospheric and low-pressure plasma have been demonstrated in many studies. The fungicidal effects of NTP can be evidenced by the killing or inactivation of fungal spores and cells in vitro (Table 1).

Table 1. Studies showing the use of NTP for fungal inactivation.

\begin{tabular}{|c|c|c|c|c|}
\hline Application & $\begin{array}{l}\text { Target Fungi } \\
\text { (Materials) }\end{array}$ & $\begin{array}{c}\text { Plasma Source } \\
\text { (Treatment Parameters) }\end{array}$ & Effects & Ref. \\
\hline & \multicolumn{4}{|c|}{ Suspension of fungal spores/cells and fungal biofilm } \\
\hline \multirow[t]{5}{*}{$\begin{array}{l}\text { Inactivation and } \\
\text { inhibition of growth }\end{array}$} & $\begin{array}{l}\text { Alternaria sp. } \\
\text { Aspergillus oryzae } \\
\text { Byssochlamys nivea } \\
\text { Cladosporium } \\
\text { sphaerospermum }\end{array}$ & $\begin{array}{l}\text { Corona discharge plasma }(9 \mathrm{kV} \text {, } \\
300 \mu \mathrm{A} \text {, filtered air) } \\
\text { Dielectric barrier discharge } \\
\text { plasma (high-voltage, air) }\end{array}$ & $\begin{array}{l}\text { C. sphaerospermum, } A \text {. sp. and } B \text {. } \\
\text { nivea, } A \text {. oryzae in order of } \\
\text { sensitivity to plasma } \\
\text { Spore inactivation time: within } \\
10-40 \text { min }\end{array}$ & [27] \\
\hline & $\begin{array}{l}\text { Ascochyta pinodella } \\
\text { Fusarium culmorum }\end{array}$ & $\begin{array}{l}\text { Dielectric barrier discharge } \\
\text { plasma }(20 \mathrm{kV}, \sim 560 \mathrm{~W} \text {, air) }\end{array}$ & $\begin{array}{l}\text { Complete inhibition of hyphal } \\
\text { growth of both fungi after } 360 \mathrm{~s} \\
\text { exposure }\end{array}$ & [13] \\
\hline & Aspergillus brasiliensis & $\begin{array}{l}\text { Plasma activated water }(\mathrm{PAW}) \text { : } \\
\text { treated with plasma jet }(1.24 \mathrm{kV} \text {, } \\
2.96 \mathrm{~A}, 3.9 \mathrm{~W} \text {, air })\end{array}$ & $\begin{array}{l}15 \% \text { reduction in spore viability } \\
\text { after } 30 \mathrm{~min} \text { in PAW }\end{array}$ & [28] \\
\hline & \multirow[t]{2}{*}{ Aspergillus flavus } & $\begin{array}{l}\text { RF plasma jet }(80-800 \mathrm{kHz} R F \\
\text { power, } 100 \mathrm{~W} \text {, mixture of argon } \\
\left.\text { Ar and oxygen } \mathrm{O}_{2}\right)\end{array}$ & $\begin{array}{l}100 \% \text { inhibition of growth } 48 \mathrm{~h} \\
\text { after } 10 \text { min treatment at } 20 \mathrm{~W}\end{array}$ & [29] \\
\hline & & $\begin{array}{l}\text { Gaseous plasma and } \\
\text { plasma-activated aqueous } \\
\text { broth (PAB) } \\
\text { Plasma source: surface barrier } \\
\text { discharge plasma ( } 5-15 \mathrm{kV} \text { at } \\
40 \mathrm{kHz}, 0.79,1.24 \text {, } \\
1.62 \mathrm{~W} / \mathrm{cm}^{2} \text {, air) }\end{array}$ & $\begin{array}{l}\text { Gaseous plasma treatment: over } \\
\text { four log reduction in spore } \\
\text { viability after } 240 \text { and } 480 \mathrm{~s} \\
\text { treatments under three power } \\
\text { conditions } \\
\text { PAB treatment: no significant } \\
\text { reduction in spore viability }\end{array}$ & [30] \\
\hline
\end{tabular}


Table 1. Cont.

\begin{tabular}{|c|c|c|c|c|}
\hline \multirow[t]{2}{*}{ Application } & $\begin{array}{l}\text { Target Fungi } \\
\text { (Materials) }\end{array}$ & $\begin{array}{c}\text { Plasma Source } \\
\text { (Treatment Parameters) }\end{array}$ & Effects & Ref. \\
\hline & \multicolumn{4}{|c|}{ Suspension of fungal spores/cells and fungal biofilm } \\
\hline & \multirow[t]{2}{*}{$\begin{array}{l}\text { Aspergillus niger } \\
\text { Penicillium citrinum }\end{array}$} & $\begin{array}{l}\text { Dielectric barrier discharge } \\
\text { plasma ( } 3 \mathrm{kV} \text { at } 230 \mathrm{~Hz} \text { on } \\
\text { dielectric ceramic electrode, } \\
-4 \mathrm{kV} \text { on needle electrode, } \\
\text { helium } \mathrm{He})\end{array}$ & $\begin{array}{l}\text { Maximum } 98-99.9 \% \\
\text { deactivation of fungal spores } \\
\text { after } 5 \mathrm{~h} \text { treatment }\end{array}$ & [31] \\
\hline & & $\begin{array}{l}\text { Microwave plasma jet }(2.45 \mathrm{GHz} \text {, } \\
1 \mathrm{~kW}, \mathrm{Ar})\end{array}$ & $\begin{array}{l}100 \% \text { inactivation of fungal } \\
\text { spores after } 1 \mathrm{~s} \text { treatment }\end{array}$ & [32] \\
\hline & $\begin{array}{l}\text { Aspergillus niger } \\
\text { Cladosporium } \\
\text { cladosporioides } \\
\text { Penicillium citrinum } \\
\text { Chaetomium sp. }\end{array}$ & $\begin{array}{l}\text { Microwave plasma jet }(2.45 \mathrm{GHz} \text {, } \\
1 \mathrm{~kW}, \mathrm{Ar})\end{array}$ & $\begin{array}{l}100 \% \text { inactivation of fungal } \\
\text { spores after } 1 \mathrm{~s} \text { treatment }\end{array}$ & [33] \\
\hline & $\begin{array}{l}\text { Aspergillus ochraceus } \\
\text { Penicillium expansum }\end{array}$ & $\begin{array}{l}\text { Plasma jet }(2.5 \mathrm{kV} \text { at } 25 \mathrm{kHz}, 3 \\
\mathrm{mA}, 4 \mathrm{~W}, \mathrm{Ar})\end{array}$ & $\begin{array}{l}\text { A. ochraceus: maximum } 3.42 \\
\log _{10} \text { CFU reduction after } 5 \mathrm{~min} \\
\text { treatment } \\
\text { P. expansum: maximum } 3.11 \\
\log _{10} \text { CFU reduction after } 5 \mathrm{~min} \\
\text { treatment }\end{array}$ & [34] \\
\hline & $\begin{array}{l}\text { Aspergillus oryzae } \\
\text { Cladosporium } \\
\text { sphaerospermum } \\
\text { Penicilium crustosum }\end{array}$ & $\begin{array}{l}\text { Corona discharge plasma }(9.7 \mathrm{kV} \text {, } \\
400 \mu \mathrm{A} \text {, filtered air })\end{array}$ & $\begin{array}{l}99.9-100 \% \text { spore inactivation } \\
\text { after } 30 \text { min treatment } \\
\text { Significant growth inhibition }\end{array}$ & {$[35]$} \\
\hline & Aureobasidium pullulans & $\begin{array}{l}\text { Dielectric barrier discharge } \\
\text { plasma }(9.3 \mathrm{kV} \text { at } 11 \mathrm{kHz}, \mathrm{Ar})\end{array}$ & $\begin{array}{l}\sim 100 \% \text { and } \sim 30 \% \text { inactivation of } \\
\text { non-melanized and melanized } \\
\text { cells after } 60 \text { min treatment } \\
\text { Improvement of fungicidal } \\
\text { efficacy of plasma by using } \\
\mathrm{FeCl}_{2} \text { and } \mathrm{FeSO}_{4} \text { together }\end{array}$ & {$[36]$} \\
\hline & Candida albicans & $\begin{array}{l}\text { Linear microdischarge plasma jet } \\
(13.56 \mathrm{MHz}, 17 \mathrm{~W}, \mathrm{He})\end{array}$ & $\begin{array}{l}\text { Changes in genome sequence, } \\
\text { enzyme activity at sublethal dose } \\
\text { No change in carbon } \\
\text { assimilation and drug } \\
\text { susceptibility at sublethal dose }\end{array}$ & [37] \\
\hline & & Plasma jet $(1.8 \mathrm{~W}, \mathrm{He})$ & $\begin{array}{l}20-30 \mathrm{~mm}^{2} \text { inhibition zone after } \\
3 \mathrm{~min} \text { treatment } \\
\text { Maximum } 11 \mathrm{~mm} \text { in diameter of } \\
\text { inhibition zone after } 3 \mathrm{~min} \\
\text { treatment on five fungal strains }\end{array}$ & {$[38,39]$} \\
\hline & & $\begin{array}{l}\text { Plasma activated water (PAW): } \\
\text { treated with nanosecond pulsed } \\
\text { DBD plasma }(50 \mathrm{~mJ} \text { per pulse, } \\
20 \mathrm{kV} \text { at } 1000 \mathrm{~Hz} \text {, air) }\end{array}$ & $\begin{array}{l}100 \% \text { cells killed after } 10-30 \mathrm{~min} \\
\text { incubation in } 5 \text { or } 10 \text { min-treated } \\
\text { PAW }\end{array}$ & {$[40]$} \\
\hline & & $\begin{array}{l}\text { RF plasma jet }(15 \mathrm{MHz} \mathrm{RF} \\
\text { power, } 10 \mathrm{kV} \text {, mixture of } 98 \% \mathrm{He} \\
\left.\text { and } 2 \% \mathrm{O}_{2}\right)\end{array}$ & $\begin{array}{l}31-82 \% \text { inhibition of growth, } \\
40-91 \% \text { reduction in ergosterol } \\
\text { biosynthesis, } 43-57 \% \text { reduction } \\
\text { in biofilm formation and } \\
\text { activities of phospholipase and } \\
\text { proteinase }\end{array}$ & {$[41]$} \\
\hline
\end{tabular}


Table 1. Cont.

Ref.

Suspension of fungal spores/cells and fungal biofilm

High-voltage nanosecond pulse plasma jet $(6 \mathrm{kV}$ at $1.5 \mathrm{kHz}$, mixture of $99 \% \mathrm{He}$ and $1 \% \mathrm{O}_{2}$

$>99.99 \%$ inactivation of fungal cells after $30-180 \mathrm{~s}$ treatment

Dielectric barrier discharge plasma $(10 \mathrm{kV}$ at $7.1 \mathrm{kHz}$, mixture of $99 \% \mathrm{He}$ and $1 \% \mathrm{O}_{2}$ )

$100 \%$ cells killed after $5 \mathrm{~min}$ treatment

$>99.9 \%$ inactivation after $3.5 \mathrm{~min}$ treatment in the condition of

Plasma jet $(8 \mathrm{kV}$ at $8 \mathrm{kHz}$, covering

mixture of $97 \% \mathrm{He}$ and $3 \% \mathrm{O}_{2}$ )

A small fraction of fungal cells inactivated even after $8 \mathrm{~min}$ treatment without covering

$100 \%$ inactivation of cells after

Dielectric barrier discharge $30 \mathrm{~s}$ treatment
Candida parapsilosis Magnusiomyces magnusii Saccharomyces cerevisiae Schizosaccharomyces pombe
Plasma jet (5-12 kV at 5-13 kHz, mixture of $99 \%$ Ar and $1 \% \mathrm{O}_{2}$ )

Colletotrichum gloeosporioides

or $99.99 \% \mathrm{O}_{2}$ )

Cordyceps bassiana

Plasma jet $(22 \mathrm{kHz}, 9 \mathrm{~W}, \mathrm{Ar})$

Electric shock-free plasma jet $(67 \mathrm{kHz}$, air)
Less than $10 \%$ survival of cells after 10 min treatment and $2 \mathrm{~h}$ incubation

Maximum $0.76 \%$ survival of $S$. pombe cells

Complete killing of fungal spores after $60 \mathrm{~s}$ treatment Disruption of membrane and leaking of cytoplasm DNA and protein damage

$96 \%$ and $56 \%$ spore inactivation after $30 \mathrm{~min}$ and $10 \mathrm{~min}$ incubation, respectively, in PAW generated using air plasma $55 \%$ and $15 \%$ spore inactivation after $30 \mathrm{~min}$ and $10 \mathrm{~min}$ incubation, respectively, in PAW generated using oxygen plasma

$16.4 \%$ spore survival after $5 \mathrm{~min}$ treatment

Reduction in DNA content and alteration to cell wall

$0.8 \%$ spore survival after $6 \mathrm{~min}$ treatment

Plasma generated $\mathrm{H}_{2} \mathrm{O}_{2}$ and

$\mathrm{NOx}$ as major players in antifungal activity

Dramatic reduction in fungal hyphal growth when $\mathrm{O}_{2}$ is used in plasma generation Fusarium oxysporum Microwave plasma jet $(2.45 \mathrm{GHz}$, $1.6 \mathrm{~W}$, mixtures of $83 \% \mathrm{Ar}$ and $17 \% \mathrm{O}_{2}, 83 \%$ Ar and $17 \% \mathrm{~N}_{2}$, or Neurospora crassa
Increased growth inhibition with length 
Table 1. Cont.

Fusarium oxysporum $\mathrm{f}$. sp. lycopersici

Dielectric barrier discharge plasma (discharge at $0.75 \mathrm{kV}$ and $80 \mathrm{~mA}, 7.5 \mathrm{~W}$, air or Ar)
$<10 \%$ survival of fungal spores after treated in saline for $10 \mathrm{~min}$ and then incubated for $6 \mathrm{~h}$ Increase in size of lipid droplets inside spore cell and number of apoptotic spores

$\sim 80 \%$ reduction in spore viability after 3 min treatment in water Less effective when treated in saline and culture media Crushed spores, cell wall

Plasma jet ( $4 \mathrm{kV}$ at $22 \mathrm{kHz}$ $13 \mathrm{~mA}, \mathrm{Ar}$ ) damage, degradation of $\beta$-carotene

Maximum 91\% spore inactivation after $9 \mathrm{~min}$ treatment and moisture was -500--1000 V and 3-16 mA $5 \mathrm{~W}$, humid air) added in working gas

Plasma generated oxygen radical source (Tough Plasma; Fuji Machine MFG Co. Ltd., Chiryu, Japan) $>2 \log _{10}$ CFU reduction in spore viability after $5 \mathrm{~min}$ treatment at distance of $10 \mathrm{~mm}$

$\sim 2 \log _{10}$ CFU reduction in spore viability after $10 \mathrm{~min}$ treatment

Microwave plasma jet $(2.45 \mathrm{GHz}$, $50 \mathrm{~W}, \mathrm{O}_{2}$ ) Decay of electron spin resonance (ESR) signal in situ from plasma treated spores

$>3 \log _{10}$ CFU reduction in spore viability after $7 \mathrm{~min}$ treatment at

Plasma jet $(6 \mathrm{kV}$ at $60 \mathrm{~Hz}, \mathrm{Ar})$ distance of $10 \mathrm{~mm}$

Plasma microdischarge torch

Penicillium sp. (discharge at 5-10 kV and $15 \mathrm{~mA}$, 7.5-15 W, air)

Inhibition of fungal growth after treatment at distance of $3 \mathrm{~cm}$ Partial spore damage after plasma treatment

$\sim 99 \%$ reduction in cell viability after 10-15 min treatment Accumulation of oxidative stress responsive transcription factor,
Dielectric barrier discharge plasma (Ar) mitochondrial fragmentation, enhanced intracellular oxidation

$\sim 100 \%$ reduction in cell viability after 2 min treatment in water Less effective when treated in

Plasma jet $(4 \mathrm{kV}$ at $22 \mathrm{kHz}$, saline and culture media $13 \mathrm{~mA}, \mathrm{Ar}$ )

Crushed cells, increased lipid

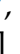
peroxidation, and DNA degradation after plasma treatment in water and saline 
Table 1. Cont.

\begin{tabular}{|c|c|c|c|c|}
\hline Application & $\begin{array}{l}\text { Target Fungi } \\
\text { (Materials) }\end{array}$ & $\begin{array}{c}\text { Plasma Source } \\
\text { (Treatment Parameters) }\end{array}$ & Effects & Ref. \\
\hline & \multicolumn{4}{|c|}{ Suspension of fungal spores/cells and fungal biofilm } \\
\hline & & $\begin{array}{l}\text { Dielectric barrier discharge } \\
\text { plasma }(12 \mathrm{kV} \text { at } 20 \mathrm{kHz}, 3.8 \mathrm{~mA} \text {, } \\
26 \mathrm{~W} \text {, air })\end{array}$ & $\begin{array}{l}\text { Maximum } \sim 2 \log _{10} \mathrm{CFU} \\
\text { reduction in cell viability after } 5 \\
\text { min treatment } \\
\text { Release of protein and nucleic } \\
\text { acids, cell cycle arrest at } \\
\text { G1 phase }\end{array}$ & [64] \\
\hline & & $\begin{array}{l}\text { Plasma microjet }(0.56 \mathrm{kV}, 30 \mathrm{~mA} \text {, } \\
\left.\text { mixture of } 98 \% \text { He and } 2 \% \mathrm{O}_{2}\right)\end{array}$ & $\begin{array}{l}\text { Maximum } 100 \% \text { reduction in cell } \\
\text { viability after } 5 \text { min treatment }\end{array}$ & [65] \\
\hline & & $\begin{array}{l}\text { Plasma microjet }(0.56 \mathrm{kV}, 30 \mathrm{~mA} \text {, } \\
\left.\text { mixture of } 98 \% \mathrm{He} \text { and } 2 \% \mathrm{O}_{2}\right)\end{array}$ & $\begin{array}{l}>2 \log _{10} \text { CFU reduction in cell } \\
\text { viability after } 5 \text { min treatment } \\
\text { in water } \\
\text { ROS and acidic } \mathrm{pH} \text { exert a } \\
\text { synergistic antimicrobial effect }\end{array}$ & [66] \\
\hline & & $\begin{array}{l}\text { Surface micro-discharge plasma } \\
\left(8 \mathrm{kV} \text { at } 8 \mathrm{kHz}, 0.07 \mathrm{~W} / \mathrm{cm}^{2}, \mathrm{He}\right)\end{array}$ & $\begin{array}{l}\text { Inactivation pattern of cells is } \\
\text { dependent on distribution and } \\
\text { concentration of } \mathrm{OH} \text { radical }\end{array}$ & [67] \\
\hline & & $\begin{array}{l}\text { Plasma microjet }(0.56 \mathrm{kV}, 30 \mathrm{~mA} \text {, } \\
\left.\text { mixture of } 98 \% \mathrm{He} \text { and } 2 \% \mathrm{O}_{2}\right)\end{array}$ & $\begin{array}{l}\text { Plasma-generated ROS leads to } \\
\text { the accumulation of intracellular } \\
\text { ROS and } \mathrm{Ca}^{2+}, \text { which in turn } \\
\text { cause apoptosis of yeast cells }\end{array}$ & [68] \\
\hline & & $\begin{array}{l}\text { Plasma microjet (discharge at } \\
0.56 \mathrm{kV} \text { and } 30 \mathrm{~mA} \text {, mixture of } \\
98 \% \mathrm{He} \text { and } 2 \% \mathrm{O}_{2} \text { ) }\end{array}$ & $\begin{array}{l}\text { Evaluated the protection effects } \\
\text { of gene manipulation and } \\
\text { reactive species scavengers } \\
\text { against plasma-induced } \\
\text { oxidative stresses: } \\
\text { overexpression of superoxide } \\
\text { dismutases reduces plasma } \\
\text { oxidative stress }\end{array}$ & [69] \\
\hline & Trichophyton rubrum & $\begin{array}{l}\text { Plasma jet }(10 \mathrm{kV} \text { at } 15 \mathrm{MHz} \text {, } \\
10 \mathrm{~W} \text {, mixture of } 98 \% \mathrm{He} \text { and } \\
\left.2 \% \mathrm{O}_{2}\right)\end{array}$ & $\begin{array}{l}\sim 91 \% \text { spore inactivation, }>50 \% \\
\text { reduction in fungal dry weight, } \\
\text { and } 53 \% \text { inhibition in ergosterol } \\
\text { synthesis after } 3 \text { min treatment }\end{array}$ & [70] \\
\hline \multirow[t]{2}{*}{$\begin{array}{l}\text { Inhibition of biofilm } \\
\text { formation }\end{array}$} & Aspergillus flavus & $\begin{array}{l}\text { Gaseous plasma and plasma } \\
\text { activated water (PAW) } \\
\text { Plasma source: dielectric barrier } \\
\text { discharge plasma }(80 \mathrm{kV} \text {, air) }\end{array}$ & $\begin{array}{l}\text { Maximum } 2.2 \text { and } 0.6 \log _{10} \text { CFU } \\
\text { reduction in spore viability after } \\
\text { treatment with gaseous plasma } \\
\text { and plasma activated } \\
\text { water, respectively } \\
\sim 50 \% \text { reduction in biofilm } \\
\text { biomass after gaseous } \\
\text { plasma treatment }\end{array}$ & [71] \\
\hline & Candida albicans & $\begin{array}{l}\text { Plasma jet }(15 \mathrm{kV} \text { at } 1 \mathrm{kHz} \text {, } \\
\text { mixture of } 99.5 \% \mathrm{He} \text { and } \\
\left.0.5 \% \mathrm{O}_{2}\right)\end{array}$ & $\begin{array}{l}\text { Reduction from } 35.6 \times 10^{2} \\
\mathrm{CFU} / \mathrm{mL} \text { to } 4.6 \times 10^{2} \mathrm{CFU} / \mathrm{mL} \\
\text { after } 8 \text { min treatment } \\
\text { of suspension } \\
\text { Complete killing of fungal cells } \\
\text { in biofilm after } 8 \text { min treatment }\end{array}$ & [72] \\
\hline
\end{tabular}


Table 1. Cont.

\begin{tabular}{|c|c|c|c|c|}
\hline Application & $\begin{array}{l}\text { Target Fungi } \\
\text { (Materials) }\end{array}$ & $\begin{array}{c}\text { Plasma Source } \\
\text { (Treatment Parameters) }\end{array}$ & Effects & Ref. \\
\hline & \multicolumn{4}{|c|}{ Suspension of fungal spores/cells and fungal biofilm } \\
\hline & & Plasma jet $(1.8 \mathrm{~W}, \mathrm{He})$ & $\begin{array}{l}40 \text { times reduction } \\
\text { in filamentation } \\
\text { Reduction in fungal adherence } \\
\text { and biofilm viability }\left(\sim 1 \log _{10}\right. \\
\text { CFU reduction in cell viability } \\
\text { within biofilm) } \\
\text { No effect on } \\
\text { exoenzyme production }\end{array}$ & [73] \\
\hline & & $\begin{array}{l}\text { Surface dielectric barrier } \\
\text { microdischarge plasma }(9 \mathrm{kV} \text { at } \\
\left.1 \mathrm{kHz}, 0.02 \mathrm{~W} / \mathrm{cm}^{2} \text {, air }\right)\end{array}$ & $\begin{array}{l}3-5 \log _{10} \text { CFU reduction in cell } \\
\text { viability within biofilm }\end{array}$ & [74] \\
\hline & & $\begin{array}{l}\text { Plasma jet (kINPen } 08 ; 2-6 \mathrm{kV} \text { at } \\
1.7 \mathrm{MHz}, 65 \mathrm{~W}, \mathrm{Ar} \text {, mixture of } \\
\left.99 \% \mathrm{Ar} \text { and } 1 \% \mathrm{O}_{2}\right)\end{array}$ & $\begin{array}{l}\text { Removal of biofilm with a } \\
\text { thickness of } 10 \text { to } 20 \mu \mathrm{m} \text { within } \\
300 \text { s plasma treatment using } \\
\text { mixture of Ar and } \mathrm{O}_{2} \text { as } \\
\text { working gas } \\
\text { Insufficient removal of biofilm } \\
\text { using Ar plasma }\end{array}$ & [75] \\
\hline & & $\begin{array}{l}\text { Plasma microjet (discharge at } \\
0.56 \mathrm{kV} \text { and } 30 \mathrm{~mA} \text {, mixture of } \\
98 \% \mathrm{He} \text { and } 2 \% \mathrm{O}_{2} \text { ) }\end{array}$ & $\begin{array}{l}\text { Complete removal of biofilms } \\
\text { after } 1 \text { min treatment } \\
\text { Severe deformation of } \\
\text { fungal elements }\end{array}$ & [76] \\
\hline & & $\begin{array}{l}\text { Plasma jet (kINPen09; } 2-6 \mathrm{kV} \text { at } \\
1.82 \mathrm{MHz}, \mathrm{Ar} \text {, mixture of } \mathrm{Ar} \\
\left.\text { and } \mathrm{O}_{2}\right) \\
\text { Hollow electrode dielectric } \\
\text { barrier discharge (HDBD) } \\
\text { plasma ( } 37.6 \mathrm{kHz} \text { RF power, } 9 \mathrm{~W} \text {, } \\
\left.9 \mathrm{kV} \text {, Ar, mixture of Ar and } \mathrm{O}_{2}\right) \\
\text { Volume dielectric barrier } \\
\text { discharge (VDBD) plasma }(10 \mathrm{kV} \\
\text { at } 40 \mathrm{kHz}, 16 \mathrm{~W}, \mathrm{Ar})\end{array}$ & $\begin{array}{l}\text { kINPen09; maximum } 1 \log _{10} \\
\text { CFU reduction in cell viability } \\
\text { within biofilm } \\
\text { HDBD; maximum } 3.3 \log _{10} \text { CFU } \\
\text { reduction in cell viability within } \\
\text { biofilm } \\
\text { VDBD; maximum } 5.2 \log _{10} \text { CFU } \\
\text { reduction in cell viability within } \\
\text { biofilm }\end{array}$ & [77] \\
\hline & \multicolumn{4}{|c|}{ Fungal contamination in agriculture and foods } \\
\hline Disinfection of seeds & $\begin{array}{l}\text { Alternaria alternata } \\
\text { Aspergillus flavus } \\
\text { Fusarium culmorum } \\
\text { (maize seeds) }\end{array}$ & $\begin{array}{l}\text { Diffuse coplanar surface barrier } \\
\text { discharge plasma } \\
\left(80 \mathrm{~W} / \mathrm{cm}^{3} \text {, air }\right)\end{array}$ & $\begin{array}{l}\text { Reduction of } 3.79 \log _{10} \mathrm{CFU} / \mathrm{g} \\
\text { in F. culmorum after } \\
60 \mathrm{~s} \text { treatment } \\
\text { Reduction of } 4.21 \log _{10} \mathrm{CFU} / \mathrm{g} \\
\text { in A. flavus and } 3.22 \log _{10} \mathrm{CFU} / \mathrm{g} \\
\text { in A. alternata after } \\
300 \mathrm{~s} \text { treatment } \\
\text { Increase in seed } \\
\text { surface wettability } \\
\text { Enhancement of seedling growth }\end{array}$ & [78] \\
\hline
\end{tabular}


Table 1. Cont.

\begin{tabular}{|c|c|c|c|c|}
\hline \multirow[t]{2}{*}{ Application } & $\begin{array}{l}\text { Target Fungi } \\
\text { (Materials) }\end{array}$ & $\begin{array}{c}\text { Plasma Source } \\
\text { (Treatment Parameters) }\end{array}$ & Effects & Ref. \\
\hline & \multicolumn{4}{|c|}{ Suspension of fungal spores/cells and fungal biofilm } \\
\hline & $\begin{array}{l}\text { Alternaria alternata } \\
\text { Alternaria botrytis } \\
\text { Aspergillus brasiliensis } \\
\text { Epicoccum nigrum } \\
\text { Fusarium culmorum } \\
\text { Fusarium poae } \\
\text { Gibberella zeae } \\
\text { Mucor hiemalis } \\
\text { Penicillium sp. } \\
\text { Rhizopus stolonifer } \\
\text { Trichoderma sp. } \\
\text { (winter wheat) }\end{array}$ & $\begin{array}{l}\text { Plane-type plasma ( } 8 \mathrm{kV} \text { at } \\
0.1-83 \mathrm{kHz} \text {, air) }\end{array}$ & $\begin{array}{l}\text { Reduction in number of fungal } \\
\text { colonies on seeds after } \\
10 \mathrm{~s} \text { treatment } \\
\text { Positive effect on seed } \\
\text { germination and initial } \\
\text { seedling development }\end{array}$ & [79] \\
\hline & $\begin{array}{l}\text { Aspergillus clavatus } \\
\text { Aspergillus flavus } \\
\text { Fusarium culmorum } \\
\text { Fusarium nivale } \\
\text { Trichothecium roseum } \\
\text { (wheat) }\end{array}$ & $\begin{array}{l}\text { Diffuse coplanar surface barrier } \\
\text { discharge plasma } \\
\left(100 \mathrm{~W} / \mathrm{cm}^{3} \text {, air }\right)\end{array}$ & $\begin{array}{l}\text { Order of efficiency in fungal } \\
\text { decrease after plasma treatment; } \\
\text { F. nivale }>\text { F. culmorum }>\text { T. roseum } \\
>\text { A. flavus }>\text { A. clavatus }\end{array}$ & [80] \\
\hline & $\begin{array}{l}\text { Aspergillus flavus } \\
\text { Aspergillus parasiticus } \\
\text { (groundnuts) }\end{array}$ & $\begin{array}{l}\text { RF plane-type plasma } \\
(13.56 \mathrm{MHz} \text { RF power, } 40 \mathrm{~W} \text { and } \\
60 \mathrm{~W} \text {, air) }\end{array}$ & $\begin{array}{l}97.9 \% \text { and } 99.3 \% \text { reduction in } \\
\text { CFU of } A \text {. parasiticus and } A . \\
\text { flavus, respectively, when treated } \\
\text { at } 60 \mathrm{~W}\end{array}$ & [81] \\
\hline & $\begin{array}{l}\text { Aspergillus niger } \\
\text { Penicillium decumbens } \\
\text { (lentil seeds) }\end{array}$ & $\begin{array}{l}\text { Diffuse coplanar surface barrier } \\
\text { discharge plasma (RPS400; } \\
400 \text { W, air) }\end{array}$ & $\begin{array}{l}1.6 \text { and } 3.1 \log _{10} \mathrm{CFU} / \mathrm{g} \\
\text { reduction for } A \text {. niger and } P \text {. } \\
\text { decumbens, respectively, after } \\
10 \text { min treatment } \\
\text { No significant effect } \\
\text { on germination }\end{array}$ & [82] \\
\hline & $\begin{array}{l}\text { Aspergillus parasiticus } \\
\text { (hazelnuts, peanuts, } \\
\text { pistachio nuts) }\end{array}$ & $\begin{array}{l}\text { Low-pressure plasma }(20 \mathrm{kV} \text { at } \\
1 \mathrm{kHz}, 300 \mathrm{~W}, 100 \text { or } 500 \mathrm{mTorr}, \\
\left.\text { air or } \mathrm{SF}_{6}\right)\end{array}$ & $\begin{array}{l}1 \text { and } 5 \log _{10} \mathrm{CFU} \text { reduction } \\
\text { after } 5 \text { min treatment with air } \\
\text { and } \mathrm{SF}_{6} \text { plasma, respectively }\end{array}$ & [83] \\
\hline & $\begin{array}{l}\text { Aspergillus sp. } \\
\text { Penicillium sp. } \\
\text { (black beans) }\end{array}$ & $\begin{array}{l}\text { Dielectric barrier discharge } \\
\text { plasma }(8 \mathrm{kV}, 510 \mathrm{~W} \text {, air })\end{array}$ & $\begin{array}{l}\text { Complete fungal disinfection } \\
\text { after treatment for at least } 10 \mathrm{~min} \\
\text { Wrinkling on seed surface and } \\
\text { change in cotyledon color }\end{array}$ & [84] \\
\hline & $\begin{array}{l}\text { (seeds of tomato, } \\
\text { wheat, bean, chickpea, } \\
\text { soybean, barley, oat, } \\
\text { rye, lentil, corn) }\end{array}$ & $\begin{array}{l}\text { Low-pressure plasma }(20 \mathrm{kV} \text { at } \\
1 \mathrm{kHz}, 300 \mathrm{~W} \text {, air or } \mathrm{SF}_{6} \text { at } \\
\text { low pressure })\end{array}$ & $\begin{array}{l}\text { Fungal decontamination below } \\
1 \% \text { of initial load } \\
3 \log _{10} \text { CFU reduction after } \\
15 \text { min treatment with } \\
\mathrm{SF}_{6} \text { plasma } \\
\text { No significant change in seed } \\
\text { germination quality }\end{array}$ & [85] \\
\hline & $\begin{array}{l}\text { Cladosporium } \\
\text { cucumerinum } \\
\text { Didymella bryoniae } \\
\text { Didymella licopersici } \\
\text { (cucumber and } \\
\text { pepper seeds) }\end{array}$ & $\begin{array}{l}\text { Surface dielectric barrier } \\
\text { discharge plasma }(20 \mathrm{kV} \text { at } \\
15 \mathrm{kHz}, 400 \mathrm{~W} \text {, air) }\end{array}$ & $\begin{array}{l}\text { No presence of } C \text {. cucumerinum } \\
\text { and } 60-80 \% \text { reduction in } D \text {. } \\
\text { bryoniae spore viability on } \\
\text { cucumber seeds after } \\
20 \mathrm{~s} \text { treatment } \\
50-80 \% \text { reduction in } D . \\
\text { lycopersici spore viability on } \\
\text { pepper seeds after } 4 \mathrm{~s} \text { treatment } \\
\text { Improvement in } \\
\text { seed germination }\end{array}$ & [86] \\
\hline
\end{tabular}


Table 1. Cont.

\section{Cladosporium fulvum} (tomato seeds)
Plasma jet $(5-12 \mathrm{kV}$ at $5-13 \mathrm{kHz}$, mixture of $99 \%$ Ar and $1 \% \mathrm{O}_{2}$ )
Maximum 14\% reduction in seed

rotting caused by fungal infection after $60 \mathrm{~s}$ treatment

Clonostachys rossmaniae

Coniochaeta fasciculat

Cylindrocarpon

destructans

Fusarium proliferatum

Humicola fuscoatra

Mortierella hyalina

Pyrenochaeta sp.

(ginseng seeds)

Dielectric barrier discharge plasma $(120 \mathrm{~V}$ at $60 \mathrm{~Hz}$, Ar or mixture of $80 \% \mathrm{Ar}$ and $20 \% \mathrm{O}_{2}$ )
$27.7 \%$ and $40 \%$ survival of fungal spores on seeds after $\mathrm{Ar}$ and $\mathrm{Ar} / \mathrm{O}_{2}$ plasma treatments, respectively

\section{Contaminated fungi (Pak-Choi seeds)}

\section{Corona discharge plasma jet} (20 kV at $58 \mathrm{kHz}, 1.5 \mathrm{~A}$, air)
1.3-2.1 $\log _{10} \mathrm{CFU} / \mathrm{g}$ reduction after 3 min treatment Positive effect on seed germination after treatment for up to $2 \mathrm{~min}$

$\sim 30 \%$ reduction in number of seeds naturally contaminated with fungi after $300 \mathrm{~s}$ treatment

(sweet basil seeds)

Surface dielectric barrier discharge plasma $(8.6 \mathrm{kV}$ at $500 \mathrm{~Hz}, 6.5 \mathrm{~W}$, air)

Improvement in growth of seedlings

$1.5 \log _{10} \mathrm{CFU} / \mathrm{g}$ reduction of natural fungal flora on seeds after 3 min treatment Positive effect on seed germination and seedling growth after treatment for up to $2 \mathrm{~min}$

Complete removal of fungal contamination from seeds after 1 min treatment and then incubation for 14 days discharge plasma $(\sim 14 \mathrm{kV}$ at $\sim 700 \mathrm{~Hz}$, air) seed germination

Barley: $25 \%$ reduction in fungal contamination on seeds after 20 min treatment, retardation of seed germination and no influence on seedling growth

(barley and corn seeds)

Glow discharge low pressure plasma (100 or $200 \mathrm{~W}, 15 \mathrm{~Pa}$, air) Corn: $\sim 40 \%$ reduction in fungal contamination on seeds after 10 min treatment, no influence on seed germination and slight improvement of seedling growth

\begin{tabular}{|c|c|c|c|}
\hline $\begin{array}{l}\text { Diaporthe/Phomopsis } \\
\text { (D/P) complex } \\
\text { (soybean seeds) }\end{array}$ & $\begin{array}{l}\text { Dielectric barrier discharge } \\
\text { plasma }(65 \text { or } 85 \mathrm{~W}, 50 \mathrm{~Hz}, \\
\left.\sim 50 \mathrm{~mA}, \mathrm{~N}_{2} \text { or } \mathrm{O}_{2}\right)\end{array}$ & $\begin{array}{l}\sim 49-81 \% \text { disinfection of seeds } \\
\text { Significant stimulating effects on } \\
\text { seed germination and vigor }\end{array}$ & [93] \\
\hline $\begin{array}{l}\text { Fusarium circinatum } \\
\text { (pine seeds) }\end{array}$ & $\begin{array}{l}\text { Diffuse coplanar surface barrier } \\
\text { discharge plasma ( } 10 \mathrm{kV} \text { at } \\
14 \mathrm{kHz}, 400 \mathrm{~W} \text {, air) }\end{array}$ & $\begin{array}{l}14-100 \% \text { disinfection of seeds } \\
\text { after treatment up to } 300 \mathrm{~s} \\
\text { Reduction in seed } \\
\text { germination percentage }\end{array}$ & [94] \\
\hline
\end{tabular}


Table 1. Cont.

\begin{tabular}{|c|c|c|c|c|}
\hline Application & $\begin{array}{l}\text { Target Fungi } \\
\text { (Materials) }\end{array}$ & $\begin{array}{c}\text { Plasma Source } \\
\text { (Treatment Parameters) }\end{array}$ & Effects & Ref. \\
\hline & \multicolumn{4}{|c|}{ Suspension of fungal spores/cells and fungal biofilm } \\
\hline & \multirow[t]{3}{*}{$\begin{array}{l}\text { Fusarium fujikuroi } \\
\text { (rice seeds) }\end{array}$} & $\begin{array}{l}\text { Plasma jet ( } 20 \mathrm{kV} \text { at } 10 \mathrm{kHz} \text {, } \\
\text { humid air) }\end{array}$ & $\begin{array}{l}\text { Reduction to } 7.8 \% \text { of non-treated } \\
\text { control in the percentage of } \\
\text { plants with disease symptoms } \\
\text { after } 10 \text { min treatment of seeds } \\
\text { in water } \\
\text { No adverse effect on seed } \\
\text { germination and } \\
\text { seedling growth }\end{array}$ & [95] \\
\hline & & $\begin{array}{l}\text { Underwater arc discharge } \\
\text { plasma }(10 \mathrm{kV} \text { at } 12 \mathrm{~Hz} \text {, air } \\
\text { in water) }\end{array}$ & $\begin{array}{l}\sim 80 \% \text { disinfection of seeds after } \\
20 \text { min treatment }\end{array}$ & [96] \\
\hline & & $\begin{array}{l}\text { Dielectric barrier discharge } \\
\text { plasma ( } 30 \mathrm{kV} \text { at } 22 \mathrm{kHz} \text {, air) }\end{array}$ & $\begin{array}{l}>92 \% \text { disinfection of seeds after } \\
120 \mathrm{~s} \text { treatment } \\
\text { Significant reduction in disease } \\
\text { development after } 10 \mathrm{~min} \\
\text { treatment on seeds } \\
\text { No adverse effect on seed } \\
\text { germination and } \\
\text { seedling growth }\end{array}$ & [97] \\
\hline & $\begin{array}{l}\text { Fusarium oxysporum } \\
\text { (Scots pine seeds) }\end{array}$ & $\begin{array}{l}\text { Diffuse coplanar surface barrier } \\
\text { discharge plasma }(20 \mathrm{kV} \text { at } \\
14 \mathrm{kHz}, 400 \mathrm{~W} \text {, air) }\end{array}$ & $\begin{array}{l}\sim 6 \% \text { disinfection of seeds after } 3 \\
\mathrm{~s} \text { treatment } \\
\text { Slight increase in seed } \\
\text { germination percentage }\end{array}$ & [98] \\
\hline & $\begin{array}{l}\text { Penicillium verrucosum } \\
\text { (wheat and } \\
\text { barley seeds) }\end{array}$ & $\begin{array}{l}\text { Dielectric barrier discharge } \\
\text { plasma }(80 \mathrm{kV} \text { at } 50 \mathrm{~Hz} \text {, air })\end{array}$ & $\begin{array}{l}\text { Maximum } 2.1 \text { and } 2.5 \log _{10} \\
\text { CFU/g reduction in barley and } \\
\text { wheat seeds, respectively, after } \\
20 \text { min treatment followed by } \\
\text { incubation for } 24 \mathrm{~h} \\
\text { No significant effect on } \\
\text { seed germination }\end{array}$ & [99] \\
\hline & $\begin{array}{l}\text { Rhizoctonia solani } \\
\text { (brassicaceous seeds) }\end{array}$ & $\begin{array}{l}\text { Atmospheric pressure plasma } \\
(10 \mathrm{kV} \text { at } 10 \mathrm{kHz}, \mathrm{Ar}) \\
\text { Low pressure plasma }(5.5 \mathrm{kV} \text { at } \\
10 \mathrm{kHz}, 80 \text { torr, Ar) }\end{array}$ & $\begin{array}{l}\text { Atmospheric-pressure plasma: } \\
97 \% \text { reduction in fungal survival } \\
\text { on seeds after } 10 \text { min treatment, } \\
\text { delay of seed germination } \\
\text { Low-pressure plasma: } 81 \% \\
\text { reduction in fungal survival on } \\
\text { seeds after } 10 \text { min treatment, no } \\
\text { change in seed germination rate }\end{array}$ & [100] \\
\hline $\begin{array}{l}\text { Disinfection of } \\
\text { post-harvest vegetables } \\
\text { and fruits }\end{array}$ & $\begin{array}{l}\text { Aspergillus flavus } \\
\text { Aspergillus parasiticus } \\
\text { (hazelnut) }\end{array}$ & $\begin{array}{l}\text { Atmospheric pressure plasma jet } \\
\left(25 \mathrm{kHz}, 655 \mathrm{~W} \text {, air or } \mathrm{N}_{2}\right) \\
\text { Low pressure RF plasma } \\
(13.56 \mathrm{MHz} \text { RF power, } 100 \mathrm{~W}, \\
\left.0.25 \mathrm{mbarr} \text {, air, } \mathrm{N}_{2} \text { or } \mathrm{O}_{2}\right)\end{array}$ & $\begin{array}{l}\text { Atmospheric-pressure plasma: } \\
5.5 \text { and } 5.4 \log _{10} \text { CFU /g } \\
\text { reduction in A. parasiticus and } A \text {. } \\
\text { flavus on hazelnuts, respectively, } \\
\text { after } 1.7 \text { min treatment } \\
\text { Low-pressure plasma: } 5.6 \text { and } \\
4.7 \log _{10} \text { CFU /g reduction in } A \text {. } \\
\text { parasiticus and A. flavus on } \\
\text { hazelnuts, respectively, after } \\
30 \text { min treatment }\end{array}$ & [101] \\
\hline
\end{tabular}


Table 1. Cont.

Ref.

Maximum 4.09-4.19 and 4.17-4.50 $\log _{10} \mathrm{CFU} / \mathrm{g}$ reduction in A. parasiticus and A. flavus on hazelnuts, respectively, after 5 min treatment with air plasma, no or little fungal regrowth

Fluidized bed plasma (5-10 kV at $18-25 \mathrm{kHz}, 655 \mathrm{~W}$, air or $\mathrm{N}_{2}$ )

during storage for 30 days

Maximum 5.20 and $5.48 \log _{10}$

$\mathrm{CFU} / \mathrm{g}$ reduction in $A$. parasiticus and A. flavus on maize, respectively, after $5 \mathrm{~min}$ treatment with air plasma, no fungal regrowth during storage for 30 days

Partial inactivation of $A$. niger Reduction in water activity Enhancement of extractability of (black pepper, allspice Microwave plasma $(2.45 \mathrm{GHz}$ $600 \mathrm{~W}, \mathrm{Ar})$ phenolics or piperine from black pepper

Complete removal of fungal spores on fruit discs after $7.5 \mathrm{~min}$ treatment with Ar flow

(date palm fruit discs) Plasma jet $(25 \mathrm{kV}$ at $\sim 25 \mathrm{kHz}, \mathrm{Ar})$ $3.5 \mathrm{~L} / \mathrm{min}$

Aspergillus niger Penicillium italicum (fruit washwater)

Plasma jet $(25 \mathrm{kV}$ at $\sim 25 \mathrm{kHz}$, Ar)

Surface dielectric barrier

Aspergillus oryzae

Penicillium digitatum (rice, lemon) discharge $(7-10 \mathrm{kV}$ at $10 \mathrm{kHz}$, air)

$74.7-100 \%$ removal of fungal contamination in the washwater of cherries after $7.5 \mathrm{~min}$ treatment $\sim 90 \%$ and $\sim 100 \%$ removal of fungal contamination on rice and lemon surface, respectively, after 20 min treatment

Inhibition of native microbial growth and natural decay of blueberries after plasma treatment Maximum $\sim 40 \%$ reduction in decay incidence in blueberries inoculated with $B$. cinerea after 20 min treatment and

Botrytis cinerea
(blueberry)

discharge plasma $(4 \mathrm{kV}$ at $8 \mathrm{kHz}$ $5 \mathrm{~W}$, air) 10-day storage

Minor effects on blueberry quality after less than $15 \mathrm{~min}$ treatment but severe oxidative damage to the blueberry peels after 20 min treatment

$>50 \%$ reduction in number of infected fruits after $5 \mathrm{~min}$ treatment in earlier days Pre-treatment of fruits by plasma before inoculation enhances the resistance to infections 
Table 1. Cont.

Colletotrichum gloeosporioides (mango) (blueberry)

(mung bean sprout)

Plasma-activated water (PAW) treated with plasma jet $(5 \mathrm{kV}$ at $40 \mathrm{kHz}, 750 \mathrm{~W}$, air)
Significant inhibition of

mycelium growth

Significant delay in disease development in mango after 7 min treatment during storage for 12 days at $30^{\circ} \mathrm{C}$

$25.8 \%$ decrease in fungal contamination on blueberry and $5.2 \%$ blueberry decay rate after 10 min treatment during storage for 20 days

$2.84 \log _{10} \mathrm{CFU} / \mathrm{g}$ reduction in yeasts and molds on mung bean after 30 min treatment in PAW No significant change in total phenolic and flavonoid contents and sensory characteristics of mung bean

0.77-1.57 $\log _{10} \mathrm{CFU} / \mathrm{g}$ reduction in yeasts and molds on kumquat after 2 min treatment

No significant change in taste, $(8 \mathrm{kV}$ at $20 \mathrm{kHz}$, air total acceptance

$0.5 \log _{10}$ CFU reduction in fungi

Plasma activated water (PAW):

(button mushroom) treated with plasma jet $(18 \mathrm{kV}$ at $10 \mathrm{kHz}$, mixture of $98 \% \mathrm{Ar}$ and $2 \% \mathrm{O}_{2}$ )

(blueberry) Plasma jet $(47 \mathrm{kHz}, 549 \mathrm{~W}$, air)

\begin{tabular}{ll}
\hline & High-field plasma system $(2 \mathrm{kV}$ \\
(banana, grape) & at $500 \mathrm{~Hz}, 20-30 \mu \mathrm{A}, 3-4 \times$ \\
& $10^{6} \mathrm{~V} / \mathrm{m}$ electric field, air $)$
\end{tabular}
on mushroom during storage for over 7 days Delay in mushroom softening No significant change in color, $\mathrm{pH}$, antioxidant properties

$1.5-2.0 \log _{10} \mathrm{CFU} / \mathrm{g}$ reduction in yeasts and molds on blueberries after 7 days

Significant reduction in firmness and anthocyanin content after treatment for over $60 \mathrm{~s}$

No increase in mold load on surface of fruits during storage in high-filed plasma system Lower amount of ethylene gas emitted during storage in high-field plasma system

$50 \%$ inhibition of fungal growth on paprika after $90 \mathrm{~s}$ treatment

Fusarium oxysporum (paprika)

Plasma jet (28 kHz, 1000 W, air)
Penicillium digitatum (citrus)
Dielectric barrier discharge plasma (10 kV at $10 \mathrm{kHz}$, air)
No significant change in color and hardness during 14 days of storage

$\sim 90 \%$ and $\sim 99 \%$ reduction in CFU number of fungal spores on citrus surface after $1 \mathrm{~s}$ and $3 \mathrm{~s}$ treatments, respectively 
Table 1. Cont.

\begin{tabular}{|c|c|c|c|c|}
\hline Application & $\begin{array}{l}\text { Target Fungi } \\
\text { (Materials) }\end{array}$ & $\begin{array}{c}\text { Plasma Source } \\
\text { (Treatment Parameters) }\end{array}$ & Effects & Ref. \\
\hline & \multicolumn{4}{|c|}{ Suspension of fungal spores/cells and fungal biofilm } \\
\hline & $\begin{array}{l}\text { Penicillium italium } \\
\text { (mandarin) }\end{array}$ & $\begin{array}{l}\text { Microwave plasma jet }(2.45 \mathrm{GHz} \text {, } \\
\left.900 \mathrm{~W}, 500-30,000 \mathrm{~Pa}, \mathrm{~N}_{2}\right)\end{array}$ & $\begin{array}{l}84 \% \text { reduction in disease } \\
\text { incidence after } 10 \text { min treatment } \\
\text { Significant increase in total } \\
\text { phenolic content and antioxidant } \\
\text { activity of mandarin peel }\end{array}$ & [119] \\
\hline & $\begin{array}{l}\text { Penicillium venetum } \\
\text { (citrus) }\end{array}$ & $\begin{array}{l}\text { Roller conveyor type dielectric } \\
\text { barrier discharge plasma } \\
(11.87 \mathrm{kV} \text { at } 8.85 \mathrm{kHz} \text {, air })\end{array}$ & $\begin{array}{l}\sim 0.7-1 \log _{10} \mathrm{CFU} / \mathrm{mL} \text { reduction } \\
\text { in viable spore number after } \\
2 \text { min treatment }\end{array}$ & [120] \\
\hline \multirow[t]{2}{*}{$\begin{array}{l}\text { Disinfection of } \\
\text { pre-harvest plants }\end{array}$} & $\begin{array}{l}\text { Botrytis cinerea } \\
\text { (cannabis } \\
\text { influorescence) }\end{array}$ & $\begin{array}{l}\text { RF plasma }(6 \mathrm{kV} \text {, low pressure } \\
\text { air with the addition of } \mathrm{H}_{2} \mathrm{O}_{2} \\
(35 \%))\end{array}$ & $\begin{array}{l}5 \log _{10} \text { CFU reduction in viable } \\
\text { fungal spores on influorescence }\end{array}$ & [121] \\
\hline & $\begin{array}{l}\text { Colletotrichum } \\
\text { gloeosporioides } \\
\text { (Green Emerald leaves) }\end{array}$ & $\begin{array}{l}\text { Plasma jet }(5 \mathrm{kV}, 11 \mathrm{~W} \text {, mixture } \\
\left.\text { of } 97 \% \text { He and } 3 \% \mathrm{O}_{2}\right)\end{array}$ & $\begin{array}{l}\text { Complete recovery of leaves } \\
\text { with black spot diameter of } \\
<2 \mathrm{~mm} \text { after plasma treatment } \\
\text { for } 3 \text { weeks (twice a day and } 10 \mathrm{~s} \\
\text { per each treatment) }\end{array}$ & [122] \\
\hline \multirow[t]{6}{*}{ Food sanitation } & $\begin{array}{l}\text { Aspergillus brasiliensis } \\
\text { (oninon powder) }\end{array}$ & $\begin{array}{l}\text { Microwave plasma } \\
(2.45 \mathrm{GHz}, 900 \mathrm{~W}, \mathrm{He})\end{array}$ & $\begin{array}{l}1.6 \log _{10} \mathrm{CFU} / \mathrm{cm}^{2} \text { reduction } \\
\text { after } 40 \mathrm{~min} \text { treatment at } 400 \mathrm{~W}\end{array}$ & [123] \\
\hline & $\begin{array}{l}\text { Aspergillus flavus } \\
\text { (in-package beef jerky) }\end{array}$ & $\begin{array}{l}\text { Flexible thin-layer plasma } \\
(15 \mathrm{kHz} \text {, air) }\end{array}$ & $\begin{array}{l}2-3 \log _{10} \text { CFU/g reduction in } \\
\text { number of viable fungal spores } \\
\text { on beef jerky after } \\
10 \text { min treatment } \\
\text { No significant change in } \\
\text { metmyoglobin content, shear } \\
\text { force, myofibrillar } \\
\text { gragmentation index } \\
\text { Negative effects on flavor, } \\
\text { off-color, and } \\
\text { overall acceptability }\end{array}$ & [124] \\
\hline & (in-package pistachio) & $\begin{array}{l}\text { Dielectric barrier discharge } \\
\text { plasma }(12.5 \mathrm{kHz} \text { suppressed by } \\
\text { a modulated pulsed signal at } \\
110 \mathrm{~Hz}, 2.49 \mathrm{~W} / \mathrm{cm}^{3} \text {, air) }\end{array}$ & $\begin{array}{l}4 \log _{10} \mathrm{CFU} / \text { sample reduction } \\
\text { in number of viable fungal } \\
\text { spores on pistachio after } \\
18 \text { min treatment } \\
\text { Slight reduction in moisture } \\
\text { content of pistachio and no } \\
\text { change in pH of pistachio }\end{array}$ & [125] \\
\hline & (red pepper powder) & $\begin{array}{l}\text { Microwave plasma }(2.45 \mathrm{GHz} \text {, } \\
50-1000 \mathrm{~W}, \mathrm{~N}_{2} \text {, mixture of } \mathrm{N}_{2} \\
\text { and } \mathrm{O}_{2}, \mathrm{He} \text {, or mixture of } \mathrm{He} \\
\left.\text { and } \mathrm{O}_{2}\right)\end{array}$ & $\begin{array}{l}2.5 \log _{10} \mathrm{CFU} / \mathrm{g} \text { reduction in } \\
\text { number of viable fungal spores } \\
\text { in red pepper powder after } \\
20 \text { min treatment with } \\
\mathrm{N}_{2} \text { plasma }\end{array}$ & [126] \\
\hline & (brown rice cereal bar) & $\begin{array}{l}\text { RF plasma jet }(50-600 \mathrm{kHz} R F \\
\text { power, } 0-40 \mathrm{~W}, \mathrm{Ar})\end{array}$ & $\begin{array}{l}\text { No fungal growth on cereal bars } \\
\text { for up to } 20 \text { days under } 25^{\circ} \mathrm{C} \\
\text { and } 100 \% \text { relative humidity after } \\
20 \text { min treatment at } 40 \mathrm{~W}\end{array}$ & [127] \\
\hline & $\begin{array}{l}\text { Aspergillus sp. } \\
\text { Rhizopus sp. } \\
\text { Penicillium sp. } \\
\text { (saffron) }\end{array}$ & $\begin{array}{l}\text { Low-pressure RF oxygen plasma } \\
(10-90 \mathrm{~W}, 8.5 \text { mTorr system } \\
\text { pressure, } 13.5 \text { mTorr working } \\
\left.\text { pressure, } \mathrm{O}_{2}\right)\end{array}$ & $\begin{array}{l}\text { Complete inactivation of fungi } \\
\text { after } 15 \text { min treatment at } 60 \mathrm{~W}\end{array}$ & [128] \\
\hline
\end{tabular}


Table 1. Cont.

\begin{tabular}{|c|c|c|c|c|}
\hline Application & $\begin{array}{l}\text { Target Fungi } \\
\text { (Materials) }\end{array}$ & $\begin{array}{c}\text { Plasma Source } \\
\text { (Treatment Parameters) }\end{array}$ & Effects & Ref. \\
\hline & \multicolumn{4}{|c|}{ Suspension of fungal spores/cells and fungal biofilm } \\
\hline & $\begin{array}{l}\text { Candida albicans } \\
\text { Saccharomyces cerevisiae } \\
\text { (tomato juice) }\end{array}$ & $\begin{array}{l}\text { AC gliding arc plasma }(3.8 \mathrm{kV} \text { at } \\
\left.50 \mathrm{~Hz}, 40 \mathrm{~W}, \mathrm{~N}_{2}\right)\end{array}$ & $\begin{array}{l}\sim 4 \log _{10} \mathrm{CFU} / \mathrm{g} \text { reduction in } \\
\text { fungal cell viability in tomato } \\
\text { juice after } 600 \mathrm{~s} \text { treatment } \\
\text { followed by storage for } 10 \text { days } \\
\text { No substantial change in the } \\
\text { physicochemical properties of } \\
\text { tomato juice }\end{array}$ & [129] \\
\hline & $\begin{array}{l}\text { Cladosporium } \\
\text { cladosporioides } \\
\text { Penicillium citrinum } \\
\text { (dried filefish fillets) }\end{array}$ & $\begin{array}{l}\text { Oxygen plasma (photoplasma; } \\
\text { Model InDuct, ID 60, BioZone } \\
\text { Scientific International Inc., } \\
\text { Orlando, FL, USA) }\end{array}$ & $\begin{array}{l}0.91 \text { and } 1.04 \log _{10} \mathrm{CFU} / \mathrm{g} \\
\text { reduction in number of } C \text {. } \\
\text { cladosporioides and } P \text {. citrinum on } \\
\text { fillets, respectively, after } \\
3-20 \text { min treatment } \\
\text { Increase in the level of } \\
\text { thiobarbituric acid reactive } \\
\text { substance (TBARS) and } \\
\text { decrease in overall sensory } \\
\text { acceptance after } 20 \\
\text { min treatment }\end{array}$ & [130] \\
\hline & $\begin{array}{l}\text { Contaminated fungi } \\
\text { (shredded salted } \\
\text { kimchi cabbage) }\end{array}$ & $\begin{array}{l}\text { Plasma-activated water (PAW): } \\
\text { treated with a plasma system } \\
(18 \mathrm{kV} \text { at } 14.3 \mathrm{kHz} \text {, air) }\end{array}$ & $\begin{array}{l}1.8 \log _{10} \mathrm{CFU} / \mathrm{g} \text { reduction in } \\
\text { yeasts and molds associated } \\
\text { with kimchi cabbages after } \\
\text { submerging in PAW treated with } \\
\text { plasma for } 120 \text { min } \\
\text { Combined treatment with mild } \\
\text { heating can enhance } \\
\text { fungal inactivation }\end{array}$ & [131] \\
\hline \multirow[t]{6}{*}{ Mycotoxin degradation } & $\begin{array}{l}\text { Aflatoxin } \\
\text { (hazelnuts) }\end{array}$ & $\begin{array}{l}\text { Dielectric barrier discharge } \\
\text { plasma }(100-150 \mathrm{kHz}, 0.4-2 \mathrm{~kW}, \\
\mathrm{N}_{2} \text { or mixture of } \mathrm{N}_{2} \text { and air) }\end{array}$ & $\begin{array}{l}>70 \% \text { reduction in the content of } \\
\text { total aflatoxins and aflatoxin } \mathrm{B} 1 \\
\text { on hazelnuts after } 12 \mathrm{~min} \\
\text { treatment at } 1000 \mathrm{~W}\end{array}$ & [132] \\
\hline & (groundnuts) & $\begin{array}{l}\text { RF-plane-type plasma } \\
\text { (13.56 MHz RF power, } 40 \mathrm{~W} \text { and } \\
60 \mathrm{~W} \text {, air) }\end{array}$ & $\begin{array}{l}>70 \% \text { and } 90 \% \text { reduction in the } \\
\text { content of aflatoxin } \mathrm{B} 1 \text { on } \\
\text { groundnuts after treatment for } \\
50 \mathrm{~min} \text { at } 40 \mathrm{~W} \text { and } 12 \mathrm{~min} \text { at } \\
60 \mathrm{~W} \text {, respectively }\end{array}$ & [81] \\
\hline & (hazelnuts) & $\begin{array}{l}\text { Atmospheric pressure plasma jet } \\
(25 \mathrm{kHz}, 655 \mathrm{~W} \text {, air)Low-pressure } \\
\text { RF plasma (13.56 MHz RF power, } \\
100 \mathrm{~W},<0.25 \mathrm{mbar} \text {, air) }\end{array}$ & $\begin{array}{l}72-73 \% \text { reduction in the amount } \\
\text { of aflatoxin B1 spiked on } \\
\text { hazelnuts after treatment with } \\
\text { both plasmas }\end{array}$ & [133] \\
\hline & (rice and wheat) & $\begin{array}{l}\text { Corona discharge plasma jet } \\
\text { ( } 20 \mathrm{kV} \text { at } 58 \mathrm{kHz} \text {, air) }\end{array}$ & $\begin{array}{l}45-56 \% \text { reduction in the level of } \\
\text { aflatoxin } \mathrm{B} 1 \text { on rice and wheat } \\
\text { after } 30 \text { min treatment }\end{array}$ & [134] \\
\hline & (corn kernels) & $\begin{array}{l}\text { DC surface barrier discharge } \\
\text { plasma }(0.18-0.31 \mathrm{~W} / \mathrm{cm} \text {, air })\end{array}$ & $\begin{array}{l}\text { Complete degradation of } \\
\text { aflatoxin B1 after } 480 \mathrm{~s} \text { treatment }\end{array}$ & [135] \\
\hline & $\begin{array}{l}\text { (slideglass, } \\
\text { pistachio nuts) }\end{array}$ & $\begin{array}{l}\text { Dielectric barrier discharge } \\
\text { plasma }(15 \mathrm{kV} \text { at } 20 \mathrm{kHz}, \\
130 \mathrm{~W} \text {, air })\end{array}$ & $\begin{array}{l}\text { Maximum } 64.63 \% \text { and } 52.42 \% \\
\text { reduction in the level of aflatoxin } \\
\text { B1 on slideglass and pistachio } \\
\text { nuts, respectively, after } \\
180 \text { s treatment }\end{array}$ & [136] \\
\hline
\end{tabular}


Table 1. Cont.

\begin{tabular}{|c|c|c|c|c|}
\hline \multirow[t]{2}{*}{ Application } & $\begin{array}{l}\text { Target Fungi } \\
\text { (Materials) }\end{array}$ & $\begin{array}{c}\text { Plasma Source } \\
\text { (Treatment Parameters) }\end{array}$ & Effects & Ref. \\
\hline & \multicolumn{4}{|c|}{ Suspension of fungal spores/cells and fungal biofilm } \\
\hline & $\begin{array}{l}\text { Deoxynivalenol, } \\
\text { zearalenone, enniatins, } \\
\text { fumonisin } \mathrm{B} 1, \mathrm{~T} 2 \text { toxin, } \\
\text { sterigmatocystin, } \\
\text { AAL toxin } \\
\text { (coverglass, rice } \\
\text { extracts) }\end{array}$ & $\begin{array}{l}\text { High-voltage pulsed } \\
\text { atmospheric-pressure-plasma } \\
(\sim 19 \mathrm{kV} \text { at } 17 \mathrm{kHz} \text {, air })\end{array}$ & $\begin{array}{l}\text { Complete removal of all } \\
\text { mycotoxins on coverglass after } \\
60 \text { s treatment; fumon: } \\
\text { fumonisin B1 is most sensitive } \\
\text { and sterigmatocystin is } \\
\text { most resistant } \\
\text { Various degradation rates of } \\
\text { mycotoxins in extracts of fungal } \\
\text { cultures on rice }\end{array}$ & [137] \\
\hline
\end{tabular}

\section{Fungal contamination in medicine}

\section{Candida albicans}

\section{Trichophyton}

Prevention of onychomycosis mentagrophytes

(fungal suspension, infected nail)
Dielectric barrier discharge

(5-20 kV at $1 \mathrm{~Hz}-1 \mathrm{kHz}$, air)

Complete killing of C. albicans

and T. mentagrophytes in

suspension after $12 \mathrm{~min}$ treatment at dose of 30 and

15 kPulses, respectively

$100 \times$ reduction in viable cell

number of $C$. albicans on nail after treatment at dose of 550 kPulses

Complete inactivation of all fungal species in vitro straight after plasma treatment

Trichophyton benhamiae Trichophyton interdigital Trichophyton rubrum (fungal suspension, patients with infected nails)
Negative DC corona discharge (7 kV, $150 \mu \mathrm{A}$, air)

More than $70 \%$ of

onychomycosis patients are

cured after the combined treatment of plasma and nail plate abrasion and refreshment

1 and $\sim 3 \log _{10}$ CFU reduction in viable cell number of T. rubrum infected in hoof discs after 45 min treatment with SMD plasma and 10 min treatment (infected sliced mixture of $99.5 \% \mathrm{He}$ and $0.5 \% \mathrm{O}_{2}$ ) Surface microdischarge (SMD) hoof discs) plasma (2.5 kV at $\sim 25 \mathrm{kHz}$, air) Floating electrode (FE) dielectric barrier discharge (DBD) plasma (6 $\mathrm{kV}$ at $4 \mathrm{kHz}$, air)

with FE-DBD plasma, respectively

In suspension: significant decrease in number of viable spores of all fungal species after 15 min treatment, complete killing of T. interdigitale and $T$. rubrum spores after

Arthroderma benhamiae Microsporum gypseu Trichophyton rubrum (fungal suspension in water, fungal spores on agar plates)
Positive and negative plasma (10 kV, $0.5 \mathrm{~mA}$, air) Cometary discharge plasma (10 kV at $20 \mathrm{kHz}$, air)
25 min treatment

On agar plates: complete killing of all fungal species, except $M$. gypseu, after 25 min treatment

\section{Candida albicans}

Candida glabrata

Candida krusei

(fungal suspension in water, fungal spores on sabouraud dextrose agar plates)
Plasma microjet (400 V, $35 \mathrm{~mA}$, mixture of $98 \% \mathrm{He}$ and $2 \% \mathrm{O}_{2}$ )
$>90 \%$ inactivation of fungal spores after $10 \mathrm{~min}$ on agar plates and $1 \mathrm{~min}$ in suspension 
Table 1. Cont.

\begin{tabular}{|c|c|c|c|c|}
\hline Application & $\begin{array}{l}\text { Target Fungi } \\
\text { (Materials) }\end{array}$ & $\begin{array}{c}\text { Plasma Source } \\
\text { (Treatment Parameters) }\end{array}$ & Effects & Ref. \\
\hline & \multicolumn{4}{|c|}{ Suspension of fungal spores/cells and fungal biofilm } \\
\hline & $\begin{array}{l}\text { Epidermophyton } \\
\text { floccosum } \\
\text { Microsporum canis } \\
\text { Microsporum gypseum } \\
\text { Trichophyton } \\
\text { mentagrophytes, } \\
\text { Trichophyton rubrum } \\
\text { (fungal suspension, } \\
\text { infected guinea pig) }\end{array}$ & $\begin{array}{l}\text { Plasma jet }(0.6 \mathrm{kV}, 15 \mathrm{~mA} \text {, } \\
21 \mathrm{kHz} \text {, air) in combination with } \\
\text { silver nanoparticles }\end{array}$ & $\begin{array}{l}\text { Reduction in values of minimum } \\
\text { inhibitory concentration (MIC) } \\
\text { of silver nanoparticles after the } \\
\text { combined treatment with plasma } \\
\text { Enhancement of fungal } \\
\text { mycelium permeability of } \\
\text { nanoparticles after the combined } \\
\text { treatment with plasma } \\
\text { Increase in efficiency of healing } \\
\text { and suppressing disease } \\
\text { symptoms of guinea pig skin } \\
\text { after the combined treatment of } \\
\text { nanoparticles and plasma }\end{array}$ & [143] \\
\hline $\begin{array}{l}\text { Prevention of } \\
\text { dermatophytosis }\end{array}$ & $\begin{array}{l}\text { Trichophyton } \\
\text { mentagrophytes } \\
\text { (Infected guinea pig) }\end{array}$ & $\begin{array}{l}\text { Cometary discharge plasma } \\
(5 \mathrm{kV} \text {, discharge at } 50-100 \mu \mathrm{A} \text {, } \\
\text { air })\end{array}$ & $\begin{array}{l}\text { A week shorter and milder } \\
\text { infection in guinea pigs treated } \\
\text { with plasma } \\
\text { Significant reduction in number } \\
\text { of viable fungal cells in guinea } \\
\text { pigs treated with plasma } \\
\text { No adverse effects on } \\
\text { guinea pigs }\end{array}$ & [144] \\
\hline $\begin{array}{l}\text { Prevention of oral } \\
\text { candidiasis }\end{array}$ & $\begin{array}{l}\text { Candida albicans } \\
\text { (Fungal biofilm, } \\
\text { infected mouse tung) }\end{array}$ & $\begin{array}{l}\text { Amplitude-modulated cold } \\
\text { atmospheric-pressure plasma jet } \\
(13 \mathrm{kV}, 32 \mathrm{kHz}, \mathrm{He})\end{array}$ & $\begin{array}{l}\text { Significant reduction in the } \\
\text { viability of } C \text {. albicans biofilms } \\
\text { after } 5 \text { min treatment } \\
\text { No significant difference in } \\
\text { values of CFU/tongue but } \\
\text { marked reduction in candidal } \\
\text { tissue invasion after } \\
\text { plasma treatment } \\
\text { No adverse effects on } \\
\text { mouse cells }\end{array}$ & [145] \\
\hline \multirow[t]{3}{*}{$\begin{array}{l}\text { Killing of clinical } \\
\text { fungal strains }\end{array}$} & $\begin{array}{l}\text { Candida albicans } \\
\text { Microsporum canis } \\
\text { Trichophyton interdigitale } \\
\text { Trichophyton rubrum } \\
\text { (fungal cells on agar } \\
\text { plates, dandruffs, shoes } \\
\text { from a patient with } \\
\text { chronic tinea pedis) }\end{array}$ & $\begin{array}{l}\text { Plasma jet }(1-5 \mathrm{kV} \text { and } 1.5 \mathrm{MHz} \\
\text { RF power, Ar) }\end{array}$ & $\begin{array}{l}\text { The largest growth inhibition } \\
\text { zone on C. albicans agar plate } \\
\text { and the smallest zone on } M \text {. canis } \\
\text { agar plate after } 15 \text { s treatment } \\
\text { Complete removal of viable } \\
\text { fungal elements of } T \text {. interdigitale } \\
\text { in dandruffs and contaminated } \\
\text { shoes after plasma treatment }\end{array}$ & [146] \\
\hline & $\begin{array}{l}\text { Candida albicans } \\
\text { (fungal cells on } \\
\text { agar plates) }\end{array}$ & $\begin{array}{l}\text { Glow discharge microplasma jet } \\
(1 \mathrm{kV} \text { at } 20 \mathrm{kHz}, 860 \text { Torr, } \mathrm{He})\end{array}$ & $\begin{array}{l}\text { Increase in growth inhibition } \\
\text { zone in sabouraud dextrose agar } \\
\text { plates after } 1.5 \text { min treatment }\end{array}$ & [147] \\
\hline & $\begin{array}{l}\text { Trichophyton } \\
\text { mentagrophytes } \\
\text { Trichophyton rubrum } \\
\text { (Fungal suspension, } \\
\text { infected skin model) }\end{array}$ & $\begin{array}{l}\text { Floating electrode-dielectric } \\
\text { barrier discharge plasma jet } \\
(8 \mathrm{kV}, \sim 33 \mathrm{~mA}, 49 \mathrm{~W}, \mathrm{Ar})\end{array}$ & $\begin{array}{l}\sim 96 \% \text { and } 90 \% \text { reduction in CFU } \\
\text { number of } T \text {. mentagrophytes and } \\
\text { T. rubrum, respectively, after } \\
5 \text { min treatment } \\
\text { Significant inhibition of hyphal } \\
\text { growth of both fungal species in } \\
\text { infected skin mimicking model } \\
\text { after plasma treatment }\end{array}$ & [148] \\
\hline
\end{tabular}

Although several studies demonstrate that fungi are less sensitive to NTP than bacteria [35], this issue is still controversial and requires more corroboration. NTP features 
different efficacies with respect to fungal inactivation, and this is dependent on the fungal species targeted, feeding gases, distance between the plasma device and sample, and treatment time. Among yeast-type fungi, Candida albicans (human fungal pathogen) and Saccharomyces cerevisiae (model yeast) have been targeted using various plasma sources (Table 1). Most studies have reported that NTP can efficiently inactivate yeast cells. Importantly, plasma generated in a sealed package has been reported to effectively inactivate $C$. albicans [43]. Several studies performed using S. cerevisiae provide the detailed information on NTP effects on fungal cells [66-68]. NTP-generated reactive oxygen species (ROS) caused the accumulation of intracellular ROS and calcium ions $\left(\mathrm{Ca}^{2+}\right)$ and ultimately led to cell apoptosis associated with cell cycle arrest at G1 phase through depolarization of mitochondrial membrane potential and fragmentation of nuclear DNA [68]. The apoptosis of $S$. cerevisiae cells was also observed in the treatment of yeast-contaminated water with NTP [66]. In this study, we found that singlet oxygen $\left({ }^{1} \mathrm{O}_{2}\right)$ among ROS generated in NTP-treated water contributed the most to yeast inactivation [66]. When NTP was applied to yeast cells on an agarose tissue model, the concentration of hydroxyl radical $(\cdot \mathrm{OH})$ and $\mathrm{pH}$ were critical for the inactivation efficiency, and the inactivation pattern of yeast cells followed the distribution of $\cdot \mathrm{OH}$ [67]. Studies have also shown that NTP can efficiently inactivate the spores of filamentous fungi, such as Aspergillus sp., Penicillium sp., Alternaria sp., Byssochlamys nivea, Cladosporium sphaerospermum, Cordyceps bassiana, and Neurospora crassa, which infect plants and spoil food (Table 1). Further, NTP has been shown to suppress ergosterol biosynthesis and increase keratinase activity in fungi [70]. The antifungal activity of NTP can be synergistically enhanced by using other compounds as shown in the study performed by Fukuda et al. [36]. This research group found that ferrous chloride and ferrous sulfate-from the Fenton reaction-improved the fungicidal effect of plasma against a melanized fungus., Aureobasidium pullulans [36].

NTP treatment has been reported to be associated with safety issues in some cases. Microbial strains that survive the action of the plasma are genetically and phenotypically modified, and these modified strains can be environmentally hazardous. Tyczkowska-Sieron et al. demonstrated that the $C$. albicans that survived after plasma treatment exhibited genetic variation, while not showing any significant changes in metabolism and drug susceptibility [37]. This indicates that NTP treatment is associated with a lower likelihood of generating genetically and phenotypically unfavorable strains [37]. However, more experimental data should be obtained regarding this safety issue. Ma et al. also investigated the safety issue of plasma: the protection of the nearby cells and tissues from plasma-induced oxidative stress [69]. This research group suggested that the elevation of antioxidant gene expression through genetic engineering, the creation of hypoxia condition, or the use of anticancer drugs could be more effective than the extracellular scavenging of reactive species to protect cells and tissues from plasma oxidative damage [69].

Biofilm development is a crucial virulence component for pathogenic fungi because biofilms are protected by a polymeric extracellular matrix (ECM) and are resistant to antifungal agents. NTP has been reported to successfully control the growth of $C$. albicans biofilms (Table 1). In different studies, C. albicans biofilm formation was inhibited by certain plasma sources, such as plasma jet or dielectric barrier discharges (DBD), using different gases (helium, argon, oxygen, or mixture of gases) (Table 1). Plasma treatment showed an efficiency that was more than two times better at inhibiting the colonization and formation of $C$. albicans biofilms (compared to chemical treatment methods) [74,77]. Sequential treatment with plasma and antifungal chemicals can eliminate C. albicans biofilms more effectively than individual treatments, thereby indicating a synergistic effect [76]. Recently, the prevention of the formation of Aspergillus flavus biofilms by direct (gas plasma treatment) and indirect (plasma-activated water, PAW) treatments was reported [71]. In this study, the metabolic activity and spore viability of $A$. flavus were significantly decreased, yielding a maximum reduction of $2.2 \log _{10} \mathrm{CFU} / \mathrm{mL}$ - with gas plasma treatment-and $0.6 \log _{10}$ $\mathrm{CFU} / \mathrm{mL}$ (with PAW treatment) [71]. 
The overall effects of NTP treatments on fungal biofilms are similar to those on bacterial biofilms. They include a significant decrease in cell viability, release of DNA and proteins, membrane lipid peroxidation, and breakdown of cell walls, resulting in impaired cell wall integrity and cell leakage [71,149]. Various reactive oxygen and nitrogen species-short-lived species such as hydroxyl radical $(\cdot \mathrm{OH})$, atomic oxygen $(\mathrm{O})$, superoxide $\left(\cdot \mathrm{O}_{2}{ }^{-}\right)$, and singlet oxygen $\left({ }^{1} \mathrm{O}_{2}\right)$, and long-lived species such as hydrogen peroxide $\left(\mathrm{H}_{2} \mathrm{O}_{2}\right)$, gaseous ozone $\left(\mathrm{O}_{3}\right)$, nitric oxide $(\cdot \mathrm{NO})$, nitrogen dioxide radical $\left(\cdot \mathrm{NO}_{2}\right)$, nitrite $\left(\mathrm{NO}_{2}{ }^{-}\right)$, and nitrate $\left(\mathrm{NO}_{3}{ }^{-}\right)$-generated from NTP are responsible for the antifungal effects [72]. In bacteria, hydroxyl radicals, gaseous ozone, and nitric oxide, in particular, are thought to be effective at inactivating biofilms $[150,151]$. These species may be able to play major roles in fungal biofilm eradication.

\subsection{Inactivation of Fungi in Agriculture and Foods}

Fungi often damage crop plants and spoil foods. NTP is known to inactivate fungal spores and cells in vitro (Table 1). NTP efficiently inactivates fungi associated with crops and food products, and various levels of decontamination and deactivation have been observed (Table 1).

Seeds contaminated with fungi are often subjected to NTP treatment (Table 1), resulting in the eradication of many seed-borne fungal diseases and mycotoxin contamination. Fungicide treatment is the standard method to disinfect contaminated seeds. The emergence of fungicide resistance and concerns about environmental safety have led to the assessment of NTP as an alternative tool to treat seeds. Studies have shown that NTP disinfects seeds contaminated (naturally or artificially) with fungi, and the efficiency of seed disinfection varies among seeds and fungal species (Table 1). Mravlje et al. analyzed the fungal community on RF plasma-treated buckwheat seeds and found a significant reduction in the frequency and diversity of fungal strains [152]. They also found that Alternaria and Epicoccum species were the most resistant to plasma [152]. NTP also disinfected seeds artificially inoculated with spores of phytopathogenic fungi, such as Alternaria alternata, Aspergillus flavus, Aspergillus niger, Aspergillus parasiticus, Cladosporium fulvum, Fusarium circinatum, Fusarium culmorum, Fusarium fujikuroi, Fusarium oxysporum, Penicillium decumbens, Penicillium verrucosum, and Rhizoctonia solani [46,78,82,83,85,94-100]. Although the sensitivity to the plasma was not significantly different among the fungal species, subtle differences were observed. Most of the studies involved the treatment of dry seeds with plasma and several showed differences between dry and wet seed treatments [95-97]. Rice seeds contaminated with F. fujikuroi, a pathogenic fungus that is responsible for causing rice bakanae disease, were treated with different plasma systems, such as air plasma jet, air DBD plasma, and underwater arc discharge plasma [95-97], and although the voltage of the plasma devices was different, these treatments resulted in the F. fujikuroi-contaminated rice seeds being disinfected with an efficiency of over $80 \%$, regardless of seed wetness [95-97].

Vegetables and fruits are also often targets of plasma disinfection. Fungi speed up the spoilage of products and produce mycotoxins harmful to humans and animals. Controlling fungal contamination is critical for improving the shelf-life and storage of post-harvest fresh produce, as well as food safety. Both artificially and naturally contaminated fruits and vegetables were examined after plasma decontamination [15,101-120]. Aspergillus and Penicillium species are frequently used for the artificial contamination of fruits and vegetables, and plasma is used before or after fungal inoculation. Plasma treatment eliminated fungal contamination from artificially inoculated fruits and vegetables by $50-100 \%$, depending on the plasma device, air pressure, feeding gases, treatment time, and voltage, regardless of pre-treatment or post-treatment (Table 1). Fungal regrowth was not observed after plasma treatment in many studies for at least a year. Even when fungal regrowth was observed, the level of regrowth was less than that observed in the non-treated control [15]. The wash water of fruits is often contaminated with fungal spores that may pollute the environment. Plasma treatment may help solve this problem. Ouf et al. showed that plasma decreased 
the number of fungal spores $(74.7-100 \%)$ in the wash water of cherries inoculated with Aspergillus niger and Penicillium italicum [107].

DBD plasma or plasma jet treatments effectively removed naturally occurring harmful fungi on blueberries, kumquats, bananas, and grapes $[109,111,113,115,116]$. In these studies, $25-100 \%$ fungal removal was achieved from the surface of fresh produce depending on plasma devices, electric power, treatment time, and feeding gases. Liu et al. developed a plasma equipped refrigerator. They found that bananas and grapes in the refrigerator were preserved for much longer with no elevation of fungal growth on the surface than those stored conventionally [116]. Mung bean sprouts and button mushrooms were decontaminated using plasma-treated water by 0.5 and $2.84 \log$ CFU reduction of fungi, respectively $[112,114]$. Many studies have shown that the properties of fruits and vegetables were not significantly altered by plasma treatment. However, Lacombe et al. observed a significant reduction in firmness and anthocyanins in blueberries after plasma treatment [115].

Pre-harvest plants are less frequently studied using plasma-mediated fungal disinfection than post-harvest fresh produce. When a plasma jet was directly applied to symptomatic leaves of Philodendron erubescens infected with fungi, no further symptom development occurred, and the leaves recovered from the infected state [122]. Inflorescences of medical cannabis inoculated with Botrytis cinerea were efficiently disinfected with plasma (5-log reduction in fungal spore CFU number) [121].

NTP has also been applied to the fungal decontamination of processed and packaged foods, and it effectively removed fungal spores. Spores of $A$. flavus on packaged beef jerky were inactivated with an efficiency of 2-3 log CFU/g reduction after plasma treatment [124]. A sealed package of fungal contaminated pistachios was completely decontaminated when the package was placed between laser electrodes for $18 \mathrm{~min}$ [125]. The fungal contamination of onion and red pepper powder, brown-rice cereal bars, saffron, and shredded salted kimchi cabbage was reduced by plasma or plasma-treated water. In these studies, fungal spores were successfully removed with an efficiency of 1.5-2.5 log CFU reduction or completely inactivated, and the shelf-life was extended up to 20 days [123,126-128,131]. Natural yeast contamination in freshly ground tomato juice was removed by glide-arc type plasma with a maximum $3 \log$ CFU reduction [129].

Mycotoxin-producing fungi present on agricultural products and foods are a threat to human and animal health. Complete or over 90\% degradation of mycotoxins, such as AAL (Alternaria alternata $f$. sp. Lycopersici) toxin, aflatoxin, deoxynivalenol, enniatins, fumonisin, sterigmatocystin, T2 toxin, trichothecenes, and zearalenone was observed after treatment with NTP for several minutes; the degradation rate varied depending on the chemical structure of the mycotoxin $[137,153]$. Mycotoxin removal from contaminated agricultural products and foods has been focused on aflatoxins (Table 1). Nuts and cereals contaminated with aflatoxin or aflatoxin-producing fungi were treated with plasma, with aflatoxin B1 being the most frequently targeted. A 50-90\% reduction in aflatoxin B1 was observed in plasma-treated nuts and cereals, depending on the type of nut and cereal, plasma source, and treatment time [81,132-136]. Siciliano et al. found that aflatoxin B1 was more sensitive to NTP than aflatoxin B2, G1, and G2 under various plasma treatment conditions [132]. Sen et al. compared the effects of atmospheric and low-pressure plasmas with that of gamma irradiation and found that gamma irradiation was more efficient at eradicating aflatoxin B1 itself and plasma treatment was more efficient at removing aflatoxin B1 from contaminated spiked hazelnuts [133].

Many studies have suggested that fungal decontamination by NTP could result from individual or synergistic actions of reactive oxygen species (ROS) and reactive nitrogen species (RNS) produced by the plasma. ROS and RNS from plasma may erode fungal cells through etching [154], and they react with chemical components of the fungal cell surface, leading to the degeneration of cell walls and membranes [155]. Many studies have also suggested that plasma-generated ROS and RNS could make the physicochemical properties 
of the surfaces of agricultural products unfavorable for fungi, or even directly inactivate fungal spores $[156,157]$.

\subsection{Inactivation of Fungi in Medicine}

Fungi cause many health problems in humans, notably skin and mucosal infections and allergies. There are approximately 300 pathogenic fungi, also known as medical fungi [158]. Most fungal infections occur in immunocompromised patients in hospitals [159]. Fungal inactivation is essential to prevent cross-infection and the further deterioration of patient health. NTP can kill bacteria and fungi in the air and decompose harmful gases and tiny particles. Therefore, it is used regularly for air disinfection in hospitals [160]. NTP is also used for the disinfection and sterilization of temperature-sensitive medical instruments and fungi-infected tissues [161,162]. NTP is frequently used in fungal skin infections. The commonly targeted fungi for NTP treatment are Trychophyton sp., Candida albicans, and Microsporum sp. (Table 1). Arthroderma benhamiae and Epidermophyton floccosum are occasionally used in experiments (Table 1). Many studies have demonstrated that Trichophyton sp., the fungal species that causes onychomycosis, was eradicated in liquid suspension or on agar media after treatment with certain NTP sources $[138,139,141,146,148]$. The maximum reduction of Trychophyton rubrum in nails was $6 \log$ when treated with a floating electrode DBD plasma [140]. In this study, authors found that the rate of decontamination of T. rubrum was faster compared to that of bacteria Escherichia coli using the same plasma device [140]. However, plasma jet and surface micro-discharge plasma were more efficient at removing E. coli than T. rubrum from infected nails [140]. Ali et al. showed that growth of T. rubrum and Trychophyton mentagrophytes was significantly inhibited in an infected skin model after treatment with a floating electrode DBD plasma [148].

Candidiasis is an infection caused by yeast-type fungi (Candida sp.) and usually affects the mouth, genitals, skin, and internal organs. Candida cells are highly susceptible to NTP, as demonstrated by in vitro treatment studies [138,142,145-147]. Depending on electric plasma pulses, nails infected with C. albicans showed a $10 \times$ and $100 \times$ reduction in fungal viability [138]. Borges et al. observed that $C$. albicans biofilms were significantly eradicated after plasma jet treatment, but not in the infected tongue [145]. However, a histological analysis showed that Candida tissue invasion was markedly reduced in plasma-treated samples [145].

A higher level of fungal decontamination occurs with the combination of NTP with other treatment methods. Lux et al. reported that nail plate abrasion, refreshment, and NTP improved fungal removal by $85.7 \%$ [139]. The combined treatment with silver nanoparticles and NTP jet decreased the minimum inhibitory concentration of silver nanoparticles [143]. NTP can be combined with other drugs to kill live fungi of some skin diseases, such as body moss and chronic wounds [163].

\section{Activation of Beneficial Fungi by NTP}

NTP is also a new technology for exerting activation effects on many organisms, such as enhancing seed germination and seedling growth, increasing antioxidant enzyme activity, elevating soluble protein and demethylation levels, accelerating wound healing processes, and activating stem cell differentiation $[26,164,165]$. Compared to the inactivation effect of NTP, the activation of cellular processes in microorganisms, including fungi, has rarely been studied. Many microorganisms are beneficial to humans and used in food, agriculture, medicine, industry, and bioremediation [166,167]. Fungi have also demonstrated their usefulness to humans [2]. Studies have shown that NTP enhanced the functional aspects of beneficial fungi through non-mutational or mutational changes (Table 2). 
Table 2. Studies showing the use of NTP for fungal activation.

\begin{tabular}{|c|c|c|c|c|}
\hline Application & Fungi & $\begin{array}{c}\text { Plasma Source } \\
\text { (Treatment Parameters) }\end{array}$ & Effects & Ref. \\
\hline \multirow[t]{3}{*}{$\begin{array}{l}\text { Enhancement } \\
\text { of spore } \\
\text { germination and pro- } \\
\text { tein secretion }\end{array}$} & Aspergillus oryzae & $\begin{array}{l}\text { Micro-dielectric barrier } \\
\text { discharge plasma }(1.2 \mathrm{kV}, \\
50-63 \mathrm{~mA}, 28.8 \mathrm{~ms} \text { on and } \\
\left.160 \mathrm{~ms} \text { off pulse times, } \mathrm{N}_{2}\right)\end{array}$ & $\begin{array}{l}\text { Significant increase in } \\
\text { percentage of spore germination } \\
\text { in phosphate buffered saline } \\
\text { (PBS) and potato dextrose broth } \\
\text { (PDB) after } 2 \text { min and } 5 \text { min } \\
\text { treatments, respectively } \\
7.4-9.3 \% \text { increase in activity of } \\
\alpha \text {-amylase in PDB after } 24 \text { and } \\
48 \text { h of plasma treatment ( } 5 \text { min) }\end{array}$ & {$[20,168]$} \\
\hline & & $\begin{array}{l}\text { Plasma jet }(\sim 0.68 \mathrm{kv} \text { at } \sim 83 \mathrm{kHz} \text {, } \\
\sim 77 \mathrm{~mA} \text {, air })\end{array}$ & $\begin{array}{l}\sim 10 \% \text { increase in spore } \\
\text { germination after } 5 \text { min and } \\
10 \text { min treatments } \\
\text { Significant elevation of } \\
\alpha \text {-amylase activity in PDB after } \\
24-96 \text { h of plasma treatment (10 } \\
\text { and } 15 \text { min) }\end{array}$ & [169] \\
\hline & Pichia pastoris & $\begin{array}{l}\text { Plasma jet }(0-15 \mathrm{kV} \text { at } \\
10 \mathrm{kHz}, \mathrm{He})\end{array}$ & $\begin{array}{l}\text { Increased production of } \\
\text { recombinant phytase by } P \text {. } \\
\text { pastoris after plasma treatment } \\
125 \% \text { increase in activity of } \\
\text { phytase in commercial enzyme } \\
\text { solution after plasma treatment }\end{array}$ & [18] \\
\hline
\end{tabular}

Atmospheric and room temperature plasma (ARTP) mutation system: radio-frequency atmospheric-pressure glow Mutagenesis Aspergillus nidulans discharge (RF APGD) plasma jet (150-300 V, 15-50 MHz RF power, $40-120 \mathrm{~W}, \mathrm{He}$ )

Commercial product from Siqingyuan Biotechnology Co., Ltd., Beijing or Wuxi, China)

\begin{tabular}{|c|c|c|c|}
\hline Aspergillus niger & ARTP mutation system & $\begin{array}{l}\text { Four mutants: gluconate } \\
\text { production of } 15.5 \%, 32.8 \% \text {, } \\
12.1 \% \text {, and } 70 \% \text { higher than that } \\
\text { of the parental strain }\end{array}$ & {$[171,172]$} \\
\hline Aspergillus oryzae & ARTP mutation system & $\begin{array}{l}\text { Mutants: } 54.7,17.3 \text {, and } 8.5 \% \\
\text { increase in activities of acid } \\
\text { protease, neutral protease, and } \\
\text { total protease, respectively, } \\
292.3 \% \text { increase in kojic acid } \\
\text { production, enhanced activities } \\
\text { of salt-tolerant proteases }\end{array}$ & $\begin{array}{c}{[19,173,} \\
174]\end{array}$ \\
\hline Aspergillus terreus & ARTP mutation system & $\begin{array}{l}\text { Mutant: growth and secretion of } \\
\text { itaconic acid in undetoxified } \\
\text { enzymatic hydrolysate }\end{array}$ & {$[175]$} \\
\hline $\begin{array}{l}\text { Auerobasidium } \\
\text { pullulans }\end{array}$ & ARTP mutation system & $\begin{array}{l}\text { Mutant: } 13.8 \% \text { increase in } \\
\text { polymalic acid production }\end{array}$ & {$[176]$} \\
\hline
\end{tabular}

Mutant: echinocandin B production of 1.3-fold higher than that of the parental strain 
Table 2. Cont.

\section{Application}

Fungi

Plasma Source

(Treatment Parameters)

Effects

Ref.

Mutant: 55\% increase in

lycopene production and

Blakeslea trispora ARTP mutation system

requirement of $10 \%$ less (than

that of parent strain) dissolved

oxygen for

maximum production

\begin{tabular}{|c|c|c|c|}
\hline Candida glabrata & ARTP mutation system & $\begin{array}{l}\text { Mutant: } 32.2-35.4 \% \text { increase in } \\
\text { pyruvate production }\end{array}$ & {$[178,179]$} \\
\hline Candida parapsilosis & ARTP mutation system & $\begin{array}{l}\text { Mutant: } 53.98 \% \text { increase in } \\
\text { D-arabitol production }\end{array}$ & [180] \\
\hline Candida tropicalis & ARTP mutation system & $\begin{array}{l}\text { Mutant: } 22 \% \text { increase in xylitol } \\
\text { production, increase in gene } \\
\text { expression and activity of } \\
\text { xylose reductase }\end{array}$ & [181] \\
\hline Fusidium coccineum & ARTP mutation system & $\begin{array}{l}\text { Mutant: } 59.4 \% \text { increase in fusidic } \\
\text { acid production }\end{array}$ & [182] \\
\hline Ganoderma lingzhi & $\begin{array}{l}\text { Dielectric barrier discharge } \\
\text { plasma }(10-15 \mathrm{kV}, \mathrm{Ar} \text { or } \mathrm{He})\end{array}$ & $\begin{array}{l}\text { Mutant: } 25.6 \% \text { increase in } \\
\text { polysaccharides production }\end{array}$ & [183] \\
\hline Glarea lozoyensis & ARTP mutation system & $\begin{array}{l}\text { Mutant: } 1.39 \text { fold increase in } \\
\text { pneumocandin } B_{0} \text { production }\end{array}$ & [184] \\
\hline Hericium erinaceus & ARTP mutation system & $\begin{array}{l}\text { Mutant: } 22 \% \text { and } 16 \% \text { increase in } \\
\text { the yield of fruiting body and } \\
\text { polysaccharide } \\
\text { production, respectively }\end{array}$ & [185] \\
\hline Mortierella alpina & ARTP mutation system & $\begin{array}{l}\text { Mutant: } 40.61 \% \text { increase in } \\
\text { arachidonic acid production }\end{array}$ & [186] \\
\hline Penicillium oxalicum & $\begin{array}{l}\text { Combined treatment with ARTP } \\
\text { mutation system and } \\
\text { ethylmethanesulfonate }\end{array}$ & $\begin{array}{l}\text { Mutant: } 61.1 \% \text { increase in } \\
\text { production of raw } \\
\text { starch-degrading enzymes }\end{array}$ & [187] \\
\hline Pichia anomala & ARTP mutation system & $\begin{array}{l}\text { Mutant: } 32.3 \% \text { increase in sugar } \\
\text { alcohol production }\end{array}$ & [188] \\
\hline
\end{tabular}

Mutants: improvement in tolerance to the inhibitory compounds in lignocellulosic hydrolysate and producing lipids with sugarcane bagasse

Rhodosporidium toruloides
ARTP mutation system hydrolysate as carbon source, improvement in production of lipids and carotenoids Enhanced expression of four genes is related to the tolerance to lignocellulosic hydrolyzate

\section{Mutant: $67 \%$ increase in} carotenoids production

Mutant: $72.54 \%$ decrease in production of methanol, which is a toxic by-product of brewing wine Mutant: $56.76 \%$ increase in
Saccharomyces cerevisiae
ARTP mutation system glutathione production, improvement of the activity of glutathione synthetases 
Table 2. Cont.

\begin{tabular}{|c|c|c|c|c|}
\hline Application & Fungi & $\begin{array}{c}\text { Plasma Source } \\
\text { (Treatment Parameters) }\end{array}$ & Effects & Ref. \\
\hline & $\begin{array}{l}\text { Sanghuangporous } \\
\text { sanghuang }\end{array}$ & ARTP mutation system & $\begin{array}{l}\text { Mutant: } 1.2-1.5 \text { fold increase in } \\
\text { polysaccharides production }\end{array}$ & {$[196]$} \\
\hline & Starmerella bombicola & ARTP mutation system & $\begin{array}{l}\text { Mutants: over } 30 \% \text { increase in } \\
\text { lactonic, acidic, or total } \\
\text { sophorolipid production }\end{array}$ & [197] \\
\hline & Trichoderma reesei & ARTP mutation system & $\begin{array}{l}\text { Mutant: increase in cellulase } \\
\text { production } \\
\text { Mutation in galactokinase gene } \\
\text { may be related to improvement } \\
\text { of cellulase production } \\
\text { Up-regulation of cellulase and } \\
\text { hemicellulose genes }\end{array}$ & {$[198]$} \\
\hline & Trichoderma viride & ARTP mutation system & $\begin{array}{l}\text { Mutant: } 2.18-2.61 \text { fold increase } \\
\text { in activities of cellulases } \\
\text { Mutant: } 1.97 \text { fold increase in } \\
\text { total cellulase activity }\end{array}$ & {$[199,200]$} \\
\hline & Yarrowia lipolytica & ARTP mutation system & $\begin{array}{l}\text { Mutant: } 45.4-51.8 \% \text { increase in } \\
\alpha \text {-ketoglutaric acid production } \\
\text { Mutations in genes regulating } \\
\text { mitochondrial biogenesis and } \\
\text { energy metabolism and a gene } \\
\text { associated with cell cycle control } \\
\text { are responsible for improvement } \\
\text { of } \alpha \text {-ketoglutaric } \\
\text { acid production } \\
\text { Mutant: the highest yield of } \\
\text { erythritol production }(64.8 \mathrm{~g} / \mathrm{L} \\
\text { erythritol from } 100 \mathrm{~g} / \mathrm{L} \text { glycerol) }\end{array}$ & [201-203] \\
\hline
\end{tabular}

\subsection{Activation through Non-Mutational Ways}

Approximately $82 \%$ of commercial enzymes in food industries are fungal in origin [204], and improving enzyme production in fungi is considered to be essential for many sectors. The efficiency of intracellular expression and the extracellular secretion of enzymes often becomes a technical bottleneck for the large-scale production of fungal enzymes. Several studies have shown that NTP can improve enzyme production in fungi (Table 2). An NTP jet using helium was used to increase the production of recombinant phytase in yeast (Pichia pastoris) [18]. Plasma treatment increased the production of recombinant phytase compared to that of the control in a time-dependent manner. In addition, the plasma significantly increased phytase activity by approximately $125 \%$ after $4 \mathrm{~h}$. Presumably, the ROS from the plasma modified the protein structure and increased enzyme activity [18].

Our research group showed that spore germination and $\alpha$-amylase secretion in Aspergillus oryzae was enhanced after treatment with a micro-dielectric barrier discharge (micro-DBD) nitrogen plasma, and plasma jet $[20,168,169]$. We also found that long-lived species $\left(\mathrm{NO}_{2}{ }^{-}\right.$and $\left.\mathrm{NO}_{3}{ }^{-}\right)$produced in the media by plasma played a critical role in activating enzyme secretion from fungal hyphae.

\subsection{Activation through Mutagenesis}

Studies have demonstrated that NTP can induce mutations in fungal genomes, improving fungal vitality and functions (Table 2). The "atmospheric and room temperature plasma" (ARTP) mutation system has been actively used for inducing fungal mutations [205]. In the ARTP mutation system, a radio-frequency atmospheric-pressure glow discharge (RF APGD) plasma jet is used, and this plasma produces a high concentration of active, neutral, and 
charged species under atmospheric pressure using radio frequency power. These species can damage the DNA strands in fungal cells, causing mutations (missense, deletion, or frame shift) through an incomplete process of gene repair [205]. Fungal spores treated with ARTP are cultured, and viable colonies are selected and cultured for generations. Colonies showing improved functions or phenotypes are selected continuously for generations as mutants. Although mutations induced by NTP may be non-usable and risky by-products in some applications, they may be helpful in the strain improvement of beneficial fungi [206].

Several studies (Table 2) have demonstrated that plasma mutagenesis has improved enzyme activities in fungi. Mutant strains of Trichoderma viride and T. reesei generated by ARTP exhibited an increase in cellulase activity of approximately a twofold [198-200]. The B-2 mutant strain of $A$. oryzae showed increased acid protease, neutral protease, and total protease activities at levels of $54.7,17.3$, and $8.5 \%$, respectively [174]. The mutant H8 of $A$. oryzae showed a significant increase in the activities of neutral proteases, alkaline proteases, and aspartyl aminopeptidase during fermentation [19]. ARTP-induced mutants of A. niger and S. cerevisiae showed improved production of glucoamylase ( $70 \%$ increase) and glutathione synthetases activity (41-72\% increase), respectively [172,195]. Similarly, mutants of P. oxalicum generated by combined ARTP/EMS mutagenesis revealed a higher production of raw starch-degrading enzymes (61.1\% increase) [187].

Mutant strains of yeast-type fungi generated using ARTP demonstrated improved biodiesel and sugar-alcohol production (Table 2). An R. toruloides (oleaginous yeast) mutant generated by ARTP showed enhanced tolerance to inhibitors in lignocellulosic hydrolysate. It grew in lignocellulosic hydrolysate and transformed carbohydrates into long-chain fatty acids, thus contributing to biodiesel production $[189,190]$. This mutant strain elevated the expression level of genes involved in regulating tolerance to stress from lignocellulosic hydrolysate [191]. Several studies showed that the production of sugar alcohols, which are useful in the food, chemical, and pharmaceutical industries, could be increased in ARTPinduced fungal mutants. For example, the ARTP-induced P. anomala mutant produced $32.3 \%$ more sugar-alcohol than the parent strain [188]. The M53 mutant of Y. lipolytica showed an increase in erythritol production from $145.2 \mathrm{~g} / \mathrm{L}$ to $200 \mathrm{~g} / \mathrm{L}$ [203]. The mutagenesis of Candida tropicalis by ARTP increased xylitol yield by $22 \%$ and enhanced xylose reductase's activity and relative gene expression [181]. The mutant A6 of C. parapsilosis showed an increase in the yield of D-arabitol ( $32.92 \mathrm{~g} / \mathrm{L})$ by $53.98 \%$ compared to the parent strain [180].

ARTP mutagenesis (Table 2) improved the production of fungal carotenoid, an important bioactive compound used as an anticancer agent, antioxidant, and immune-response stimulant. The production of the carotenoid lycopene was $55 \%$ higher in the Blakeslea trispora mutant A5 than in the parent strain [177]. The combined use of chemical and ARTP mutagenesis showed increased levels of carotenoids and lipids in the R. toruloides XR-2 mutant strain [192]. The K4 mutant strain of $R$. mucilaginosa generated from the same method produced a $121 \%$ higher concentration of carotenoids than the original strain [193].

An improved production of organic and fatty acids was found in ARTP-induced fungal mutants. For example, the 1-C6 mutant strain of $Y$. lipolytica produced a significantly higher amount of $\alpha$-ketoglutaric acid than the wild type (51.8\% higher in $500 \mathrm{~mL}$ shake flasks and $45.4 \%$ higher in a $3 \mathrm{~L}$ fermenter) [201]. A combined mutagenesis with ARTP and diethyl sulfate of Mortierella alpine produced the D20 mutant that exhibited 40.61\% increased yields of arachidonic acid (ARA), and increased the yield of total fatty acids by about 7\% [186]. Polymalic acid (PMA) produced from the ARTP-induced Auerobasidium pullulans AH-21 mutant was $13.8 \%$ higher than that produced by the wild type [176]. The ARTP-induced A. terreus mutant AT-90 produced the highest level of itaconic acid [175]. In an $A$. oryzae mutant strain generated by a combined mutagenesis of microwave, UV irradiation, heat-LiCl, and ARTP kojic acid was quantified as approximately $47-292.3 \%$ higher than the original strain, and the transcription of the genes related to kojic acid biosynthesis was also enhanced [173]. A transcriptome analysis of Fusidium coccineum and its ARTP-mutagenized strains showed that the transcription levels of most genes involved in fusidic acid biosynthesis significantly increased in the mutant strain, leading 
to the enhanced production of fusidic acid [182]. Luo et al. discovered an ARTP-induced mutant of C. glabrata that showed a 32.2\% increase in pyruvate levels [178]. Improved polysaccharide production was also reported in medicinal fungi. The polysaccharide content of Ganoderma lingzhi was increased $25.6 \%$ by ARTP-induced mutants [183]. The yield of fruiting body and polysaccharide in an ARTP-induced mutant of H. erinaceus increased by $22 \%$ and $16 \%$, respectively [185]. Similarly, in mutants of S. sanghuang, polysaccharide yields were significantly increased by 1.2 to 1.5 fold [196].

The improved production of organic compounds in fungi by ARTP has been reported. For example, gluconate production in $A$. niger mutant strains was enhanced by $12.1-32.8 \%$ [171]. The yield of pneumocandin B0, a starting molecule for the semi-synthesis of the antifungal drug, echinocandin, was elevated in G. lozoyensis mutants by 1.39 to 1.65 fold [184]. Echinocandin B production in A. nidulans was also improved by ARTP mutagenesis with a 1.3 fold increase [170]. ARTP mutants of S. bombicola enhanced the production of sophorolipids (SLs) used in several applications, such as food, cosmetics, detergent, environmental, petroleum nanotechnology, and pharmaceutical industries [197]. Specific and total SL production in S. bombicola mutant strains exhibited an increase of over $30 \%$ in lactonic SLs, acidic SLs, and total SL production compared with the wild strain [197]. ARTP mutagenesis was useful in reducing the production of highly toxic methanol by $S$. cerevisiae in brewed wine [194]. The S. cerevisiae S12 mutant decreased methanol production by $72.54 \%$ [194].

NTP is a useful tool for increasing the production of enzymes and many useful metabolites and compounds in beneficial fungi. However, several factors, such as the type of plasma and fungi, the dosage of plasma, and the RONS (reactive oxygen and nitrogen species) released, are important considerations when evaluating the effects of plasma on the activation of fungi. The majority of current studies focus on using NTP as a mutagenesis tool. Few studies have examined NTP for generating activation effects on fungal cellular processes without causing mutations. Further research is required to show whether the activation effects on fungi are due to mutations or some other cause.

\section{Mechanisms of Fungal Inactivation and Activation by NTP}

Many studies have suggested that short- and long-lived reactive species generated by NTP are the main factors that regulate the inactivation and activation of microorganisms, including fungi $[18,20,156]$. NTP, with an influx of air on liquid surfaces, can generate reactive species, such as free electrons, $\cdot \mathrm{O}_{2}{ }^{-}, \cdot \mathrm{H}, \cdot \mathrm{OH}, \cdot \mathrm{NO}, \cdot \mathrm{NO}_{2}, \mathrm{O}_{3}$, atomic oxygen $(\mathrm{O})$, and singlet oxygen $\left({ }^{1} \mathrm{O}_{2}\right)$, which feature relatively short lifetimes [207]. Short-lifetime species produced on liquid surfaces react with species in solution, producing secondary species, such as hydrogen peroxide $\left(\mathrm{H}_{2} \mathrm{O}_{2}\right)$, nitrite $\left(\mathrm{NO}_{2}{ }^{-}\right)$, and nitrate $\left(\mathrm{NO}_{3}{ }^{-}\right)$, which can exert a more substantial influence on cells and organisms [208]. Short- and long-lifetime species are responsible for the interaction between plasma and biological objects, and double-edged effects (inactivation and activation) of NTP may result mainly from the action of reactive species (Figure 2). Although the mechanisms underlying the action of NTP are more often reported for bacteria than for fungi, it is assumed that many of the mechanisms may be common [156]. 


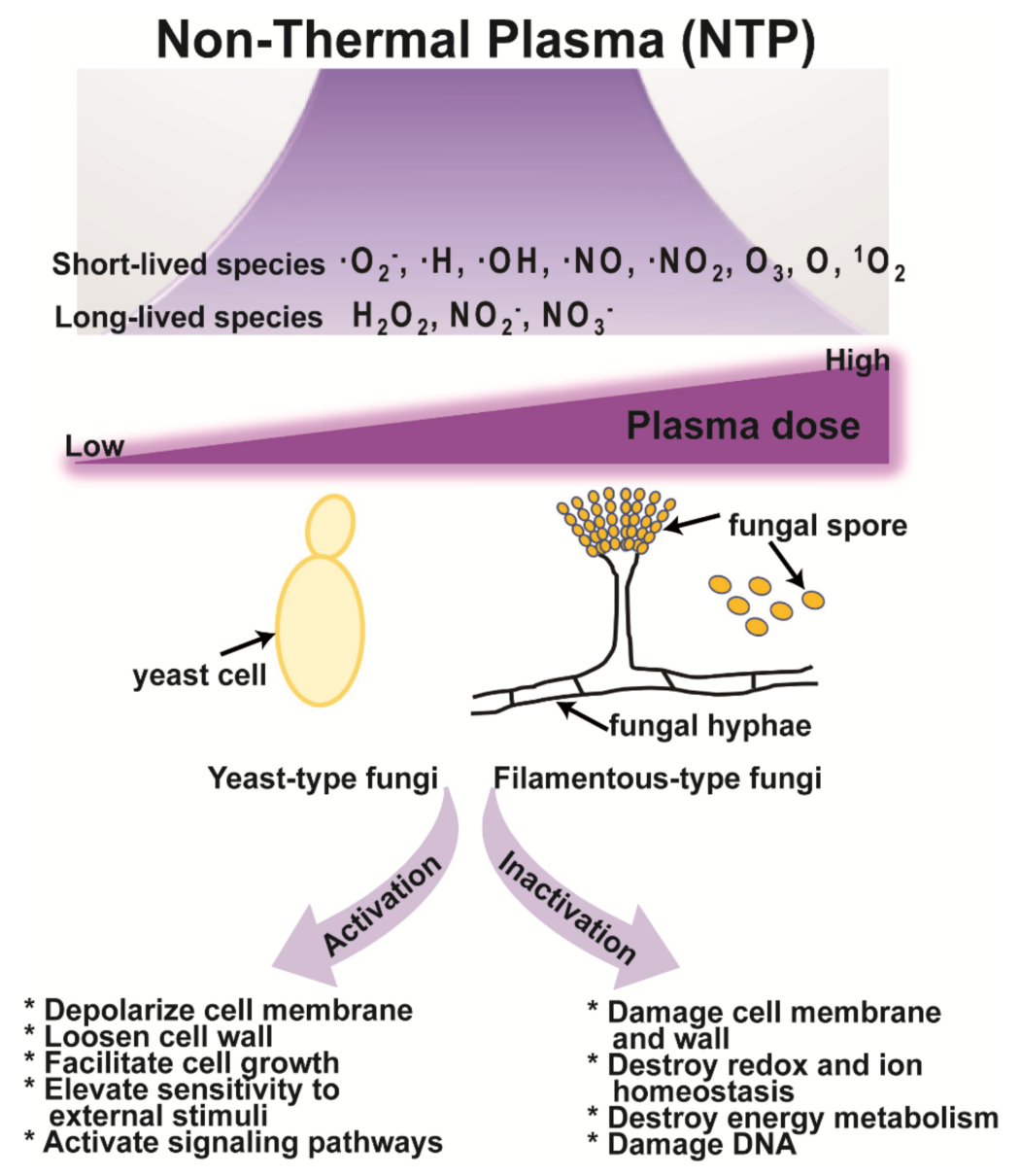

Figure 2. Proposed mechanism of fungal activation and inactivation by NTP.

NTP may destroy cell membranes, intracellular redox, ion homeostasis (intracellular $\mathrm{H}^{+}$and $\mathrm{K}^{+}$), and energy metabolism (mitochondrial membrane potential, intracellular $\mathrm{Ca}^{2+}$, and ATP levels), and damage the DNA (Figure 2). Plasma-generated reactive species interact with proteins to form superoxides or interact with DNA to cause DNA alkylation or inter-chain cross-linking, as well as changing the cell's metabolic activity and genetic characteristics $[209,210]$. In yeast, inactivation by NTP produces $\cdot \mathrm{OH}$ and ${ }^{1} \mathrm{O}_{2}$. The $\cdot \mathrm{OH}$ radical attacks cell membranes and increases the permeability, while the ${ }^{1} \mathrm{O}_{2}$ radical interferes with cell metabolism [211]. Other studies have found that free radicals damaged the cell membranes and walls and could enter cells where they inhibited the normal physiological activities of DNA, RNA, and proteins, eventually killing the microorganisms [212].

The mechanisms of fungal activation by NTP have been rarely studied. NTP can trigger the depolarization of the cell membrane, elevation of calcium influx, and enhancement of secretory vesicle accumulation near the hyphal tips resulting in increased enzyme secretion in a fungus (Figure 2) [20]. In bacteria, charged reactive species generated by NTP penetrate the cell wall under the action of an electric field and make the cell wall looser. This facilitates bacterial cell growth, increases its sensitivity to external stimuli, and activates signaling pathways in bacterial cells [213]. However, the strong and continuous action of charged species on a bacterium can result in the degeneration of the cell membrane, the outflow of the cell lysate, and the death of the bacterium [213]. Similar processes can occur in fungi if the intensity of the plasma is not continuously strong.

\section{Conclusions and Future Perspectives}

Fungi exert a significant impact on human life as agents threatening human health and the ecosystem or by providing benefits to industry. The efficient control and use of fungal resources are advantageous for the economy and industry. Studies performed 
over the past decade demonstrate that NTP offers great potential as a universal tool for inactivating harmful fungi or activating the functions of beneficial fungi. An enormous amount of data support that NTP can be an efficient and eco-friendly remover of fungi without marked damage on the quality of contaminated and infected objects, thereby replacing chemical fungicides. However, further research is still needed to fine-tune the conditions of NTP to support optimal fungal control on foods and agricultural products and restrain human and animal fungal pathogens. Safety issues related to NTP treatment, such as the generation of genetically and phenotypically modified unfavorable fungal strains, as well as the protection of nearby cells and tissues from plasma treatment, also require further detailed investigations.

A limited number of studies are available on the application of NTP to activating the functional aspects of beneficial fungi. The majority of these studies are focused on using NTP to generate functionally improved mutant strains. Enhancing the functional aspects of fungi without mutations may make NTP a safe and reliable technology. Therefore, future research should focus on addressing this aspect of NTP. In addition, the application of NTP technology to improving the functions of beneficial fungi could create a potential emerging, low-competition market in the food and agriculture industries.

For the productive application of NTP technology, the establishment of a database of fungal responses to various plasma intensities may be essential because a broad spectrum of effects can be obtained based on the different doses or intensities of NTP used.

Funding: This work was funded by the National Research Foundation of Korea (NRF) (No. 2020R1F1A1070942 and 2021R1A6A1A03038785) and the Excellent Researcher Support Project of Kwangwoon University in 2021.

Institutional Review Board Statement: Not applicable.

Informed Consent Statement: Not applicable.

Data Availability Statement: Not applicable.

Conflicts of Interest: The authors declare no conflict of interest.

\section{References}

1. Purvis, A.; Hector, A. Getting the Measure of Biodiversity. Nature 2000, 405, 212-219. [CrossRef] [PubMed]

2. Hyde, K.D.; Xu, J.; Rapior, S.; Jeewon, R.; Lumyong, S.; Niego, A.G.T.; Abeywickrama, P.D.; Aluthmuhandiram, J.V.S.; Brahamanage, R.S.; Brooks, S.; et al. The Amazing Potential of Fungi: 50 Ways We Can Exploit Fungi Industrially. Fungal Divers. 2019, 97, 1-136. [CrossRef]

3. Wu, B.; Hussain, M.; Zhang, W.; Stadler, M.; Liu, X.; Xiang, M. Current Insights into Fungal Species Diversity and Perspective on Naming the Environmental DNA Sequences of Fungi. Mycology 2019, 10, 127-140. [CrossRef] [PubMed]

4. Fisher, M.C.; Gow, N.A.R.; Gurr, S.J. Tackling Emerging Fungal Threats to Animal Health, Food Security and Ecosystem Resilience. Philos. Trans. R. Soc. Lond. B Biol. Sci. 2016, 371, 20160332. [CrossRef]

5. Bongomin, F.; Gago, S.; Oladele, R.O.; Denning, D.W. Global and Multi-National Prevalence of Fungal Diseases-Estimate Precision. J. Fungi 2017, 3, 57. [CrossRef]

6. Benedict, K.; Jackson, B.R.; Chiller, T.; Beer, K.D. Estimation of Direct Healthcare Costs of Fungal Diseases in the United States. Clin. Infect. Dis. 2019, 68, 1791-1797. [CrossRef] [PubMed]

7. Konopka, J.; Casadevall, A.; Taylor, J.; Heitman, J.; Cowen, L. One Health: Fungal Pathogens of Humans, Animals, and Plants; Report on an American Academy of Microbiology Colloquium, Washington DC USA, 18 October 2017; America Society for Microbiology: Washington, DC, USA, 2019.

8. Cairns, T.C.; Nai, C.; Meyer, V. How a Fungus Shapes Biotechnology: 100 Years of Aspergillus niger Research. Fungal Biol. Biotechnol. 2018, 5, 13. [CrossRef]

9. McKelvey, S.M.; Murphy, R.A. Biotechnological Use of Fungal Enzymes; John Wiley \& Sons, Inc.: Hoboken, NJ, USA, 2017; pp. 201-225.

10. Meyer, V.; Andersen, M.R.; Brakhage, A.A.; Braus, G.H.; Caddick, M.X.; Cairns, T.C.; de Vries, R.P.; Haarmann, T.; Hansen, K.; Hertz-Fowler, C.; et al. Current Challenges of Research on Filamentous Fungi in Relation to Human Welfare and a Sustainable Bio-Economy: A White Paper. Fungal Biol. Biotechnol. 2016, 3, 6. [CrossRef] [PubMed]

11. Brandt, M.E.; Park, B.J. Think Fungus-Prevention and Control of Fungal Infections. Emerg. Infect. Dis. 2013, 19, 1688-1689. [CrossRef] 
12. Kersey, P.J.; Collemare, J.; Cockel, C.; Das, D.; Dulloo, E.M.; Kelly, L.J.; Lettice, E.; Malécot, V.; Maxted, N.; Metheringham, C.; et al. Selecting for Useful Properties of Plants and Fungi-Novel Approaches, Opportunities, and Challenges. Plants People Planet 2020, 2, 409-420. [CrossRef]

13. Avramidis, G.; Stüwe, B.; Wascher, R.; Bellmann, M.; Wieneke, S.; von Tiedemann, A.; Viöl, W. Fungicidal Effects of an Atmospheric Pressure Gas Discharge and Degradation Mechanisms. Surf. Coatings Technol. 2010, 205, S405-S408. [CrossRef]

14. Xiong, Z.; Lu, X.P.; Feng, A.; Pan, Y.; Ostrikov, K. Highly Effective Fungal Inactivation in He+ O2 Atmospheric-Pressure Nonequilibrium Plasmas. Phys. Plasmas 2010, 17, 123502. [CrossRef]

15. Ambrico, P.F.; Šimek, M.; Rotolo, C.; Morano, M.; Minafra, A.; Ambrico, M.; Pollastro, S.; Gerin, D.; Faretra, F.; De Miccolis Angelini, R.M. Surface Dielectric Barrier Discharge Plasma: A Suitable Measure Against Fungal Plant Pathogens. Sci. Rep. 2020 10, 3673. [CrossRef] [PubMed]

16. Schnabel, U.; Yarova, K.; Zessin, B.; Stachowiak, J.; Ehlbeck, J. The Combination of Plasma-Processed Air (PPA) and Plasma-Treated Water (PTW) Causes Synergistic Inactivation of Candida albicans SC5314. Appl. Sci. 2020, 10, 3303. [CrossRef]

17. Shimada, K.; Takashima, K.; Kimura, Y.; Nihei, K.; Konishi, H.; Kaneko, T. Humidification Effect of Air Plasma Effluent Gas on Suppressing Conidium Germination of a Plant Pathogenic Fungus in the Liquid Phase. Plasma Process. Polym. 2020, 17, 1900004. [CrossRef]

18. Farasat, M.; Arjmand, S.; Ranaei Siadat, S.O.; Sefidbakht, Y.; Ghomi, H. The Effect of Non-Thermal Atmospheric Plasma on the Production and Activity of Recombinant Phytase Enzyme. Sci. Rep. 2018, 8, 16647. [CrossRef]

19. Gao, X.; Liu, E.; Yin, Y.; Yang, L.; Huang, Q.; Chen, S.; Ho, C.T. Enhancing Activities of Salt-Tolerant Proteases Secreted by Aspergillus oryzae Using Atmospheric and Room-Temperature Plasma Mutagenesis. J. Agric. Food Chem. 2020, 68, 2757-2764. [CrossRef] [PubMed]

20. Veerana, M.; Mitra, S.; Ki, S.H.; Kim, S.M.; Choi, E.H.; Lee, T.; Park, G. Plasma-Mediated Enhancement of Enzyme Secretion in Aspergillus oryzae. Microb. Biotechnol. 2021, 14, 262-276. [CrossRef]

21. Fridman, A. Plasma Chemistry; Cambridge University Press: Cambridge, UK, 2008.

22. Domonkos, M.; Tichá, P.; Trejbal, J.; Demo, P. Applications of Cold Atmospheric Pressure Plasma Technology in Medicine, Agriculture and Food Industry. Appl. Sci. 2021, 11, 4809. [CrossRef]

23. Chen, Z.; Xu, R.G.; Chen, P.; Wang, Q. Potential Agricultural and Biomedical Applications of Cold Atmospheric Plasma-Activated Liquids with Self-Organized Patterns Formed at the Interface. IEEE Trans. Plasma Sci. 2020, 48, 3455-3471. [CrossRef]

24. Mijatović, S.; Savić-Radojević, A.; Plješa-Ercegovac, M.; Simić, T.; Nicoletti, F.; Maksimović-Ivanić, D. The Double-Faced Role of Nitric Oxide and Reactive Oxygen Species in Solid Tumors. Antioxidants 2020, 9, 374. [CrossRef]

25. Sakudo, A.; Yagyu, Y.; Onodera, T. Disinfection and Sterilization Using Plasma Technology: Fundamentals and Future Perspectives for Biological Applications. Int. J. Mol. Sci. 2019, 20, 5216. [CrossRef]

26. Braný, D.; Dvorská, D.; Halašová, E.; Škovierová, H. Cold Atmospheric Plasma: A Powerful Tool for Modern Medicine. Int. J. Mol. Sci. 2020, 21, 2932. [CrossRef]

27. Julák, J.; Soušková, H.; Scholtz, V.; Kvasničková, E.; Savická, D.; Kř́ha, V. Comparison of Fungicidal Properties of Non-Thermal Plasma Produced by Corona Discharge and Dielectric Barrier Discharge. Folia Microbiol. 2018, 63, 63-68. [CrossRef] [PubMed]

28. Ki, S.H.; Noh, H.; Ahn, G.R.; Kim, S.H.; Kaushik, N.K.; Choi, E.H.; Lee, G.J. Influence of Nonthermal Atmospheric PlasmaActivated Water on the Structural, Optical, and Biological Properties of Aspergillus brasiliensis Spores. Appl. Sci. 2020, $10,6378$. [CrossRef]

29. Intanon, W.; Vichiansan, N.; Leksakul, K.; Boonyawan, D.; Kumla, J.; Suwannarach, N.; Lumyong, S. Inhibition of the AflatoxinProducing Fungus Aspergillus flavus by a Plasma Jet System. J. Food Process. Preserv. 2021, 45, e15045. [CrossRef]

30. Hojnik, N.; Modic, M.; Ni, Y.; Filipič, G.; Cvelbar, U.; Walsh, J.L. Effective Fungal Spore Inactivation with an Environmentally Friendly Approach Based on Atmospheric Pressure Air Plasma. Environ. Sci. Technol. 2019, 53, 1893-1904. [CrossRef] [PubMed]

31. Nojima, H.; Park, R.E.; Kwon, J.H.; Suh, I.; Jeon, J.; Ha, E.; On, H.K.; Kim, H.R.; Choi, K.; Lee, K.H.; et al. Novel Atmospheric Pressure Plasma Device Releasing Atomic Hydrogen: Reduction of Microbial-Contaminants and OH Radicals in the Air. J. Phys. D Appl. Phys. 2007, 40, 501-509. [CrossRef]

32. Park, B.J.; Lee, D.H.; Park, J.-C.; Lee, I.-S.; Lee, K.-Y.; Hyun, S.O.; Chun, M.-S.; Chung, K.-H. Sterilization Using a MicrowaveInduced Argon Plasma System at Atmospheric Pressure. Phys. Plasmas 2003, 10, 4539-4544. [CrossRef]

33. Park, J.C.; Park, B.J.; Han, D.W.; Lee, D.H.; Lee, I.S.; Hyun, S.O.; Chun, M.S.; Chung, K.H.; Aihara, M.; Takatori, K. Fungal Sterilization Using Microwave-Induced Argon Plasma at Atmospheric Pressure. J. Microbiol. Biotechnol. 2004, 14, 188-192.

34. Herceg, Z.; Režek Jambrak, A.; Vukušić, T.; Stulić, V.; Stanzer, D.; Milošević, S. The Effect of High-Power Ultrasound and Gas Phase Plasma Treatment on Aspergillus spp. and Penicillium spp. Count in Pure Culture. J. Appl. Microbiol. 2015, 118, 132-141. [CrossRef]

35. Soušková, H.; Scholtz, V.; Julák, J.; Kommová, L.; Savická, D.; Pazlarová, J. The Survival of Micromycetes and Yeasts Under the Low-Temperature Plasma Generated in Electrical Discharge. Folia microbiol. 2011, 56, 77-79. [CrossRef] [PubMed]

36. Fukuda, S.; Kawasaki, Y.; Izawa, S. Ferrous Chloride and Ferrous Sulfate Improve the Fungicidal Efficacy of Cold Atmospheric Argon Plasma on Melanized Aureobasidium pullulans. J. Biosci. Bioeng. 2019, 128, 28-32. [CrossRef] [PubMed]

37. Tyczkowska-Sieroń, E.; Kałużewski, T.; Grabiec, M.; Kałużewski, B.; Tyczkowski, J. Genotypic and Phenotypic Changes in Candida albicans as a Result of Cold Plasma Treatment. Int. J. Mol. Sci. 2020, 21, 8100. [CrossRef] [PubMed] 
38. Nishime, T.M.C.; Borges, A.C.; Koga-Ito, C.Y.; Machida, M.; Hein, L.R.O.; Kostov, K.G. Non-Thermal Atmospheric Pressure Plasma Jet Applied to Inactivation of Different Microorganisms. Surf. Coatings Technol. 2017, 312, 19-24. [CrossRef]

39. Kostov, K.G.; Borges, A.C.; Koga-Ito, C.Y.; Nishime, T.M.C.; Prysiazhnyi, V.; Honda, R.Y. Inactivation of Candida albicans by Cold Atmospheric Pressure Plasma Jet. IEEE Trans. Plasma Sci. 2015, 43, 770-775. [CrossRef]

40. Laurita, R.; Barbieri, D.; Gherardi, M.; Colombo, V.; Lukes, P. Chemical Analysis of Reactive Species and Antimicrobial Activity of Water Treated by Nanosecond Pulsed DBD Air Plasma. Clin. Plasma Med. 2015, 3, 53-61. [CrossRef]

41. Rahimi-Verki, N.; Shapoorzadeh, A.; Razzaghi-Abyaneh, M.; Atyabi, S.M.; Shams-Ghahfarokhi, M.; Jahanshiri, Z.; GholamiShabani, M. Cold Atmospheric Plasma Inhibits the Growth of Candida albicans by Affecting Ergosterol Biosynthesis and Suppresses the Fungal Virulence Factors In Vitro. Photodiagnosis Photodyn. Ther. 2016, 13, 66-72. [CrossRef]

42. Sedghizadeh, P.P.; Chen, M.; Schaudinn, C.; Gorur, A.; Jiang, C. Inactivation Kinetics Study of an Atmospheric-Pressure Cold-Plasma Jet Against Pathogenic Microorganisms. IEEE Trans. Plasma Sci. 2012, 40, 2879-2882. [CrossRef]

43. Song, Y.; Liu, D.; Ji, L.; Wang, W.; Zhao, P.; Quan, C.; Niu, J.; Zhang, X. The Inactivation of Resistant Candida albicans in a Sealed Package by Cold Atmospheric Pressure Plasmas. Plasma Processes Polym. 2012, 9, 17-21. [CrossRef]

44. Shi, X.-M.; Zhang, G.-J.; Yuan, Y.-K.; Ma, Y.; Xu, G.-M.; Yang, Y. Research on the Inactivation Effect of Low-Temperature Plasma on Candida albicans. IEEE Trans. Plasma Sci. 2008, 36, 498-503. [CrossRef]

45. Siadati, S.; Pet'ková, M.; Kenari, A.J.; Kyzek, S.; Gálová, E.; Zahoranová, A. Effect of a Non-Thermal Atmospheric Pressure Plasma Jet on Four Different Yeasts. J. Phys. D: Appl. Phys. 2021, 54, 025204. [CrossRef]

46. Lu, Q.; Liu, D.; Song, Y.; Zhou, R.; Niu, J. Inactivation of the Tomato Pathogen Cladosporium fulvum by an Atmospheric-Pressure Cold Plasma Jet. Plasma Process. Polym. 2014, 11, 1028-1036. [CrossRef]

47. Wu, M.; Liu, C.; Chiang, C.; Lin, Y.; Lin, Y.; Chang, Y.; Wu, J. Inactivation Effect of Colletotrichum gloeosporioides by Long-Lived Chemical Species Using Atmospheric-Pressure Corona Plasma-Activated Water. IEEE Trans. Plasma Sci. 2019, 47, 1100-1104. [CrossRef]

48. Kim, J.Y.; Lee, I.H.; Kim, D.; Kim, S.H.; Kwon, Y.-W.; Han, G.-H.; Cho, G.; Choi, E.H.; Lee, G.J. Effects of Reactive Oxygen Species on the Biological, Structural, and Optical Properties of Cordyceps pruinosa Spores. RSC Adv. 2016, 6, 30699-30709. [CrossRef]

49. Lee, G.J.; Sim, G.B.; Choi, E.H.; Kwon, Y.-W.; Kim, J.Y.; Jang, S.; Kim, S.H. Optical and Structural Properties of Plasma-Treated Cordyceps bassiana Spores as Studied by Circular Dichroism, Absorption, and Fluorescence Spectroscopy. J. Appl. Phys. 2015, 117, 023303. [CrossRef]

50. Noh, H.; Kim, J.E.; Kim, J.Y.; Kim, S.H.; Han, I.; Lim, J.S.; Ki, S.H.; Choi, E.H.; Lee, G.J. Spore Viability and Cell Wall Integrity of Cordyceps pruinosa Treated with an Electric Shock-Free, Atmospheric-Pressure Air Plasma Jet. Appl. Sci. 2019, 9, 3921. [CrossRef]

51. Na, Y.H.; Park, G.; Choi, E.H.; Uhm, H.S. Effects of the Physical Parameters of a Microwave Plasma Jet on the Inactivation of Fungal Spores. Thin Solid Films 2013, 547, 125-131. [CrossRef]

52. Panngom, K.; Lee, S.H.; Park, D.H.; Sim, G.B.; Kim, Y.H.; Uhm, H.S.; Park, G.; Choi, E.H. Non-Thermal Plasma Treatment Diminishes Fungal Viability and Up-Regulates Resistance Genes in a Plant Host. PLoS ONE 2014, 9, e99300. [CrossRef]

53. Lee, G.J.; Park, G.; Choi, E.H. Optical and Biological Properties of Plasma-Treated Neurospora crassa Spores as Studied by Absorption, Circular Dichroism, and Raman Spectroscopy. J. Korean Phys. Soc. 2017, 71, 670-678. [CrossRef]

54. Kang, M.H.; Hong, Y.J.; Attri, P.; Sim, G.B.; Lee, G.J.; Panngom, K.; Kwon, G.C.; Choi, E.H.; Uhm, H.S.; Park, G. Analysis of the Antimicrobial Effects of Nonthermal Plasma on Fungal Spores in Ionic Solutions. Free Radic. Biol. Med. 2014, 72, 191-199. [CrossRef] [PubMed]

55. Park, G.; Ryu, Y.H.; Hong, Y.J.; Choi, E.H.; Uhm, H.S. Cellular and Molecular Responses of Neurospora crassa to Non-Thermal Plasma at Atmospheric Pressure. Appl. Phys. Lett. 2012, 100, 063703. [CrossRef]

56. Liu, K.; Wang, C.; Hu, H.; Lei, J.; Han, L. Indirect Treatment Effects of Water-Air MHCD Jet on the Inactivation of Penicillium digitatum Suspension. IEEE Trans. Plasma Sci. 2016, 44, 2729-2737. [CrossRef]

57. Hashizume, H.; Ohta, T.; Fengdong, J.; Takeda, K.; Ishikawa, K.; Hori, M.; Ito, M. Inactivation Effects of Neutral Reactive-Oxygen Species on Penicillium digitatum Spores Using Non-Equilibrium Atmospheric-Pressure Oxygen Radical Source. Appl. Phys. Lett. 2013, 103, 153708. [CrossRef]

58. Iseki, S.; Hashizume, H.; Jia, F.; Takeda, K.; Ishikawa, K.; Ohta, T.; Ito, M.; Hori, M. Inactivation of Penicillium digitatum Spores by a High-Density Ground-State Atomic Oxygen-Radical Source Employing an Atmospheric-Pressure Plasma. Appl. Phys. Express 2011, 4, 116201. [CrossRef]

59. Ishikawa, K.; Mizuno, H.; Tanaka, H.; Tamiya, K.; Hashizume, H.; Ohta, T.; Ito, M.; Iseki, S.; Takeda, K.; Kondo, H.; et al. Real-Time In Situ Electron Spin Resonance Measurements on Fungal Spores of Penicillium digitatum During Exposure of Oxygen Plasmas. Appl. Phys. Lett. 2012, 101, 013704. [CrossRef]

60. Iseki, S.; Ohta, T.; Aomatsu, A.; Ito, M.; Kano, H.; Higashijima, Y.; Hori, M. Rapid Inactivation of Penicillium digitatum Spores Using High-Density Nonequilibrium Atmospheric Pressure Plasma. Appl. Phys. Lett. 2010, 96, 153704. [CrossRef] [PubMed]

61. Veremii, Y.; Andriiash, I.; Tsvyd, N.; Chernyak, V.Y.; Sukhomlyn, M.; Martysh, E. Influence of Cold Atmospheric Plasma of Microdischarge on Fungal Mycelium and Spores Growing. Probl. At. Sci. Technol. 2019, 119, 233-236.

62. Itooka, K.; Takahashi, K.; Izawa, S. Fluorescence Microscopic Analysis of Antifungal Effects of Cold Atmospheric Pressure Plasma in Saccharomyces cerevisiae. Appl. Microbiol. Biotechnol. 2016, 100, 9295-9304. [CrossRef]

63. Ryu, Y.H.; Kim, Y.H.; Lee, J.Y.; Shim, G.B.; Uhm, H.S.; Park, G.; Choi, E.H. Effects of Background Fluid on the Efficiency of Inactivating Yeast with Non-Thermal Atmospheric Pressure Plasma. PLoS ONE 2013, 8, e66231. 
64. Chen, H.; Bai, F.; Xiu, Z. Oxidative Stress Induced in Saccharomyces cerevisiae Exposed to Dielectric Barrier Discharge Plasma in Air at Atmospheric Pressure. IEEE Trans. Plasma Sci. 2010, 38, 1885-1891. [CrossRef]

65. Feng, H.; Wang, R.; Sun, P.; Wu, H.; Liu, Q.; Fang, J.; Zhu, W.; Li, F.; Zhang, J. A Study of Eukaryotic Response Mechanisms to Atmospheric Pressure Cold Plasma by Using Saccharomyces cerevisiae Single Gene Mutants. Appl. Phys. Lett. 2010, $97,131501$. [CrossRef]

66. Xu, H.; Ma, R.; Zhu, Y.; Du, M.; Zhang, H.; Jiao, Z. A Systematic Study of the Antimicrobial Mechanisms of Cold AtmosphericPressure Plasma for Water Disinfection. Sci. Total Environ. 2020, 703, 134965. [CrossRef] [PubMed]

67. Xu, H.; Zhu, Y.; Cui, D.; Du, M.; Wang, J.; Ma, R.; Jiao, Z. Evaluating the Roles of $\mathrm{OH}$ radicals, $\mathrm{H}_{2} \mathrm{O}_{2}, \mathrm{ORP}_{\text {and }} \mathrm{pH}$ in the Inactivation of Yeast Cells on a Tissue Model by Surface Micro-Discharge Plasma. J. Phys. D Appl. Phys. 2019, $52,395201$. [CrossRef]

68. Ma, R.N.; Feng, H.Q.; Liang, Y.D.; Zhang, Q.; Tian, Y.; Su, B.; Zhang, J.; Fang, J. An Atmospheric-Pressure Cold Plasma Leads to Apoptosis in Saccharomyces cerevisiae by Accumulating Intracellular Reactive Oxygen Species and Calcium. J. Phys. D Appl. Phys. 2013, 46, 285401. [CrossRef]

69. Ma, R.; Feng, H.; Li, F.; Liang, Y.; Zhang, Q.; Zhu, W.; Zhang, J.; Becker, K.H.; Fang, J. An Evaluation of Anti-oxidative Protection for Cells against Atmospheric Pressure Cold Plasma Treatment. Appl. Phys. Lett. 2012, 100, 123701. [CrossRef]

70. Shapourzadeh, A.; Rahimi-Verki, N.; Atyabi, S.M.; Shams-Ghahfarokhi, M.; Jahanshiri, Z.; Irani, S.; Razzaghi-Abyaneh, M. Inhibitory Effects of Cold Atmospheric Plasma on the Growth, Ergosterol Biosynthesis, and Keratinase Activity in Trichophyton rubrum. Arch. Biochem. Biophys. 2016, 608, 27-33. [CrossRef] [PubMed]

71. Los, A.; Ziuzina, D.; Boehm, D.; Cullen, P.J.; Bourke, P. Inactivation Efficacies and Mechanisms of Gas Plasma and PlasmaActivated Water Against Aspergillus flavus Spores and Biofilms: A Comparative Study. Appl. Environ. Microbiol. 2020, 86, e02619-19. [CrossRef] [PubMed]

72. He, M.; Duan, J.; Xu, J.; Ma, M.; Chai, B.; He, G.; Gan, L.; Zhang, S.; Duan, X.; Lu, X.; et al. Candida albicans Biofilm Inactivated by Cold Plasma Treatment In Vitro and In Vivo. Plasma Process. Polym. 2020, 17, 1900068. [CrossRef]

73. Borges, A.C.; Castaldelli Nishime, T.M.; Kostov, K.G.; de Morais Gouvêa Lima, G.; Lacerda Gontijo, A.V.; de Carvalho, J.N.M.M.; Yzumi Honda, R.; Yumi Koga-Ito, C. Cold Atmospheric Pressure Plasma Jet Modulates Candida Albicans Virulence Traits. Clin. Plasma Med. 2017, 7, 9-15. [CrossRef]

74. Maisch, T.; Shimizu, T.; Isbary, G.; Heinlin, J.; Karrer, S.; Klämpfl, T.G.; Li, Y.F.; Morfill, G.; Zimmermann, J.L. Contact-Free Inactivation of Candida albicans Biofilms by Cold Atmospheric Air Plasma. Appl. Environ. Microbiol. 2012, 78, 4242-4247. [CrossRef]

75. Fricke, K.; Koban, I.; Tresp, H.; Jablonowski, L.; Schröder, K.; Kramer, A.; Weltmann, K.-D.; von Woedtke, T.; Kocher, T. Atmospheric Pressure Plasma: A High-Performance Tool for the Efficient Removal of Biofilms. PLoS ONE 2012, 7, e42539. [CrossRef] [PubMed]

76. Sun, Y.; Yu, S.; Sun, P.; Wu, H.; Zhu, W.; Liu, W.; Zhang, J.; Fang, J.; Li, R. Inactivation of Candida Biofilms by Non-Thermal Plasma and Its Enhancement for Fungistatic Effect of Antifungal Drugs. PLoS ONE 2012, 7, e40629. [CrossRef] [PubMed]

77. Koban, I.; Matthes, R.; Hübner, N.-O.; Welk, A.; Meisel, P.; Holtfreter, B.; Sietmann, R.; Kindel, E.; Weltmann, K.-D.; Kramer, A.; et al. Treatment of Candida albicans Biofilms with Low-Temperature Plasma Induced by Dielectric Barrier Discharge and Atmospheric Pressure Plasma Jet. New J. Phys. 2010, 12, 073039. [CrossRef]

78. Zahoranová, A.; Hoppanová, L.; Šimončicová, J.; Tučeková, Z.; Medvecká, V.; Hudecová, D.; Kaliňáková, B.; Kováčik, D.; Černák, M. Effect of Cold Atmospheric Pressure Plasma on Maize Seeds: Enhancement of Seedlings Growth and Surface Microorganisms Inactivation. Plasma Chem. Plasma Process. 2018, 38, 969-988. [CrossRef]

79. Kordas, L.; Pusz, W.; Czapka, T.; Kacprzyk, R. The Effect of Low-Temperature Plasma on Fungus Colonization of Winter Wheat Grain and Seed Quality. Pol. J. Environ. Stud. 2015, 24, 433-438.

80. Zahoranová, A.; Henselová, M.; Hudecová, D.; Kalináková, B.; Kováčik, D.; Medvecká, V.; Černák, M. Effect of Cold Atmospheric Pressure Plasma on the Wheat Seedlings Vigor and on the Inactivation of Microorganisms on the Seeds Surface. Plasma Chem. Plasma Process. 2016, 36, 397-414. [CrossRef]

81. Devi, Y.; Thirumdas, R.; Sarangapani, C.; Deshmukh, R.R.; Annapure, U.S. Influence of Cold Plasma on Fungal Growth and Aflatoxins Production on Groundnuts. Food Control 2017, 77, 187-191. [CrossRef]

82. Waskow, A.; Betschart, J.; Butscher, D.; Oberbossel, G.; Klöti, D.; Büttner-Mainik, A.; Adamcik, J.; von Rohr, P.R.; Schuppler, M. Characterization of Efficiency and Mechanisms of Cold Atmospheric Pressure Plasma Decontamination of Seeds for Sprout Production. Front. Microbiol. 2018, 9, 3164. [CrossRef] [PubMed]

83. Basaran, P.; Basaran-Akgul, N.; Oksuz, L. Elimination of Aspergillus parasiticus from Nut Surface with Low Pressure Cold Plasma (LPCP) Treatment. Food Microbiol. 2008, 25, 626-632. [CrossRef]

84. Rüntzel, C.L.; Da Silva, J.R.; Da Silva, B.A.; Moecke, E.S.; Scussel, V.M. Effect of Cold Plasma on Black Beans (Phaseolus vulgaris L.), Fungi Inactivation and Micro-Structures Stability. Emir. J. Food Agric. 2019, 31, 864-873. [CrossRef]

85. Selcuk, M.; Oksuz, L.; Basaran, P. Decontamination of Grains and Legumes Infected with Aspergillus spp. and Penicillum spp. by Cold Plasma Treatment. Bioresour. Technol. 2008, 99, 5104-5109. [CrossRef] [PubMed]

86. Štěpánová, V.; Slavíček, P.; Kelar, J.; Prášil, J.; Smékal, M.; Stupavská, M.; Jurmanová, J.; Černák, M. Atmospheric Pressure Plasma Treatment of Agricultural Seeds of Cucumber (Cucumis sativus L.) and Pepper (Capsicum annuum L.) with Effect on Reduction of Diseases and Germination Improvement. Plasma Process. Polym. 2018, 15, e1700076. [CrossRef] 
87. Lee, Y.; Lee, Y.Y.; Kim, Y.S.; Balaraju, K.; Mok, Y.S.; Yoo, S.J.; Jeon, Y. Enhancement of Seed Germination and Microbial Disinfection on Ginseng by Cold Plasma Treatment. J. Ginseng Res. 2021, 45, 519-526. [CrossRef] [PubMed]

88. Puligundla, P.; Kim, J.W.; Mok, C. Effect of Atmospheric Pressure Plasma Treatment on Seed Decontamination and Sprouting of Pak Choi (Brassica rapa L. subsp chinensis (L.) Hanelt). Chiang Mai J. Sci. 2018, 45, 2679-2690.

89. Ambrico, P.F.; Šimek, M.; Morano, M.; De Miccolis Angelini, R.M.D.; Minafra, A.; Trotti, P.; Ambrico, M.; Prukner, V.; Faretra, F. Reduction of Microbial Contamination and Improvement of Germination of Sweet Basil (Ocimum basilicum L.) Seeds via Surface Dielectric Barrier Discharge. J. Phys. D Appl. Phys. 2017, 50, 305401. [CrossRef]

90. Kim, J.W.; Puligundla, P.; Mok, C. Effect of Corona Discharge Plasma Jet on Surface-Borne Microorganisms and Sprouting of Broccoli Seeds. J. Sci. Food Agric. 2017, 97, 128-134. [CrossRef]

91. Khamsen, N.; Onwimol, D.; Teerakawanich, N.; Dechanupaprittha, S.; Kanokbannakorn, W.; Hongesombut, K.; Srisonphan, S. Rice (Oryza sativa L.) Seed Sterilization and Germination Enhancement via Atmospheric Hybrid Nonthermal Discharge Plasma. ACS Appl. Mater. Interfaces 2016, 8, 19268-19275. [CrossRef]

92. Brasoveanu, M.; Nemţanu, M.; Carmen, S.-B.; Karaca, G.; Erper, İ. Effect of Glow Discharge Plasma on Germination and Fungal Load of Some Cereal Seeds. Rom. Rep. Phys. 2015, 67, 617-624.

93. Pérez Pizá, M.C.; Prevosto, L.; Zilli, C.; Cejas, E.; Kelly, H.; Balestrasse, K. Effects of Non-Thermal Plasmas on Seed-Borne Diaporthe/Phomopsis Complex and Germination Parameters of Soybean Seeds. Innov. Food Sci. Emerg. Technol. 2018, $49,82-91$. [CrossRef]

94. Sera, B.; Zahoranova, A.; Bujdakova, H.; Sery, M. Disinfection from Pine Seeds Contaminated with Fusarium circinatum Nirenberg \& O'Donnell Using Non-Thermal Plasma Treatment. Rom. Rep. Phys. 2019, 71, 701.

95. Ochi, A.; Konishi, H.; Ando, S.; Sato, K.; Yokoyama, K.; Tsushima, S.; Yoshida, S.; Morikawa, T.; Kaneko, T.; Takahashi, H. Management of Bakanae and Bacterial Seedling Blight Diseases in Nurseries by Irradiating Rice Seeds with Atmospheric Plasma. Plant Pathol. 2017, 66, 67-76. [CrossRef]

96. Kang, M.H.; Pengkit, A.; Choi, K.; Jeon, S.S.; Choi, H.W.; Shin, D.B.; Choi, E.H.; Uhm, H.S.; Park, G. Differential Inactivation of Fungal Spores in Water and on Seeds by Ozone and Arc Discharge Plasma. PLoS ONE 2015, 10, e0139263. [CrossRef]

97. Jo, Y.K.; Cho, J.; Tsai, T.C.; Staack, D.; Kang, M.H.; Roh, J.H.; Shin, D.B.; Cromwell, W.; Gross, D. A Non-Thermal Plasma Seed Treatment Method for Management of a Seedborne Fungal Pathogen on Rice Seed. Crop Sci. 2014, 54, 796-803. [CrossRef]

98. Śswiecimska, M.; Tulik, M.; Šerá, B.; Golińska, P.; Tomeková, J.; Medvecká, V.; Bujdáková, H.; Oszako, T.; Zahoranová, A.; Šerý, M. Non-Thermal Plasma Can Be Used in Disinfection of Scots Pine (Pinus sylvestris L.) Seeds Infected with Fusarium oxysporum. Forests 2020, 11, 837. [CrossRef]

99. Los, A.; Ziuzina, D.; Akkermans, S.; Boehm, D.; Cullen, P.J.; Van Impe, J.; Bourke, P. Improving Microbiological Safety and Quality Characteristics of Wheat and Barley by High Voltage Atmospheric Cold Plasma Closed Processing. Food Res. Int. 2018, 106, 509-521. [CrossRef] [PubMed]

100. Nishioka, T.; Takai, Y.; Kawaradani, M.; Okada, K.; Tanimoto, H.; Misawa, T.; Kusakari, S. Seed Disinfection Effect of Atmospheric Pressure Plasma and Low Pressure Plasma on Rhizoctonia solani. Biocontrol Sci. 2014, 19, 99-102. [CrossRef]

101. Sen, Y.; Onal-Ulusoy, B.; Mutlu, M. Aspergillus Decontamination in Hazelnuts: Evaluation of Atmospheric and Low-Pressure Plasma Technology. Innov. Food Sci. Emerg. Technol. 2019, 54, 235-242. [CrossRef]

102. Dasan, B.G.; Boyaci, I.H.; Mutlu, M. Nonthermal Plasma Treatment of Aspergillus spp. Spores on Hazelnuts in an Atmospheric Pressure Fluidized Bed Plasma System: Impact of Process Parameters and Surveillance of the Residual Viability of Spores. J. Food Eng. 2017, 196, 139-149. [CrossRef]

103. Dasan, B.G.; Boyaci, I.H.; Mutlu, M. Inactivation of Aflatoxigenic Fungi (Aspergillus spp.) on Granular Food Model, Maize, in an Atmospheric Pressure Fluidized Bed Plasma System. Food Control 2016, 70, 1-8. [CrossRef]

104. Dasan, B.G.; Mutlu, M.; Boyaci, I.H. Decontamination of Aspergillus flavus and Aspergillus parasiticus Spores on Hazelnuts via Atmospheric Pressure Fluidized Bed Plasma Reactor. Int. J. Food Microbiol. 2016, 216, 50-59. [CrossRef]

105. Wiktor, A.; Hrycak, B.; Jasiński, M.; Rybak, K.; Kieliszek, M.; Kraśniewska, K.; Witrowa-Rajchert, D. Impact of Atmospheric Pressure Microwave Plasma Treatment on Quality of Selected Spices. Appl. Sci. 2020, 10, 6815. [CrossRef]

106. Ouf, S.A.; Basher, A.H.; Mohamed, A.A. Inhibitory Effect of Double Atmospheric Pressure Argon Cold Plasma on Spores and Mycotoxin Production of Aspergillus niger Contaminating Date Palm Fruits. J. Sci. Food Agric. 2015, 95, 3204-3210. [CrossRef]

107. Ouf, S.A.; Mohamed, A.-A.H.; El-Sayed, W.S. Fungal Decontamination of Fleshy Fruit Water Washes by Double Atmospheric Pressure Cold Plasma. C.L.E.A.N. Soil Air Water 2016, 44, 134-142. [CrossRef]

108. Hayashi, N.; Yagyu, Y.; Yonesu, A.; Shiratani, M. Sterilization Characteristics of the Surfaces of Agricultural Products Using Active Oxygen Species Generated by Atmospheric Plasma and UV Light. Jpn. J. Appl. Phys. 2014, 53, 05FR03. [CrossRef]

109. Hu, X.; Sun, H.; Yang, X.; Cui, D.; Wang, Y.; Zhuang, J.; Wnag, X.; Ma, R.; Jiao, Z. Potential Use of Atmospheric Cold Plasma for Postharvest Preservation of Blueberries. Postharvest Biol. Technol. 2021, 179, 111564. [CrossRef]

110. Phan, K.T.K.; Phan, H.T.; Brennan, C.S.; Regenstein, J.M.; Jantanasakulwong, K.; Boonyawan, D.; Phimolsiripol, Y. Gliding Arc Discharge Non-Thermal Plasma for Retardation of Mango Anthracnose. LWT 2019, 105, 142-148. [CrossRef]

111. Dong, X.Y.; Yang, Y.L. A Novel Approach to Enhance Blueberry Quality During Storage Using Cold Plasma at Atmospheric Air Pressure. Food Bioprocess Technol. 2019, 12, 1409-1421. [CrossRef]

112. Xiang, Q.; Liu, X.; Liu, S.; Ma, Y.; Xu, C.; Bai, Y. Effect of Plasma-Activated Water on Microbial Quality and Physicochemical Characteristics of Mung Bean Sprouts. Innov. Food Sci. Emerg. Technol. 2019, 52, 49-56. [CrossRef] 
113. Puligundla, P.; Lee, T.; Mok, C. Effect of Intermittent Corona Discharge Plasma Treatment for Improving Microbial Quality and Shelf Life of Kumquat (Citrus japonica) Fruits. LWT 2018, 91, 8-13. [CrossRef]

114. Xu, Y.; Tian, Y.; Ma, R.; Liu, Q.; Zhang, J. Effect of Plasma Activated Water on the Postharvest Quality of Button Mushrooms, Agaricus bisporus. Food Chem. 2016, 197, 436-444. [CrossRef] [PubMed]

115. Lacombe, A.; Niemira, B.A.; Gurtler, J.B.; Fan, X.; Sites, J.; Boyd, G.; Chen, H. Atmospheric Cold Plasma Inactivation of Aerobic Microorganisms on Blueberries and Effects on Quality Attributes. Food Microbiol. 2015, 46, 479-484. [CrossRef]

116. Liu, C.-M.; Nishida, Y.; Iwasaki, K.; Ting, K. Prolonged Preservation and Sterilization of Fresh Plants in Controlled Environments Using High-Field Plasma. IEEE Trans. Plasma Sci. 2011, 39, 717-724. [CrossRef]

117. Go, S.-M.; Park, M.-R.; Kim, H.-S.; Choi, W.S.; Jeong, R.-D. Antifungal Effect of Non-Thermal Atmospheric Plasma and Its Application for Control of Postharvest Fusarium oxysporum Decay of Paprika. Food Control 2019, 98, 245-252. [CrossRef]

118. Yagyu, Y.; Hatayama, Y.; Hayashi, N.; Mishima, T.; Nishioka, T.; Sakudo, A.; Ihara, T.; Ohshima, T.; Kawasaki, H.; Suda, Y. Direct Plasma Disinfection of Green Mold Spore on Citrus by Atmospheric Pressure Dielectric Barrier Discharge for Agricultural Applications. Trans. Mat. Res. Soc. Japan 2016, 41, 127-130. [CrossRef]

119. Won, M.Y.; Lee, S.J.; Min, S.C. Mandarin Preservation by Microwave-Powered Cold Plasma Treatment. Innov. Food Sci. Emerg. Technol. 2017, 39, 25-32. [CrossRef]

120. Sakudo, A.; Yagyu, Y. Application of a Roller Conveyor Type Plasma Disinfection Device with Fungus-Contaminated Citrus Fruits. AMB Express 2021, 11, 16. [CrossRef]

121. Jerushalmi, S.; Maymon, M.; Dombrovsky, A.; Freeman, S. Effects of Cold Plasma, Gamma and e-Beam Irradiations on Reduction of Fungal Colony Forming Unit Levels in Medical Cannabis Inflorescences. J. Cannabis Res. 2020, 2, 12. [CrossRef]

122. Zhang, X.; Liu, D.; Zhou, R.; Song, Y.; Sun, Y.; Zhang, Q.; Niu, J.; Fan, H.; Yang, S.-Z. Atmospheric Cold Plasma Jet for Plant Disease Treatment. Appl. Phys. Lett. 2014, 104, 043702. [CrossRef]

123. Kim, J.E.; Oh, Y.J.; Won, M.Y.; Lee, K.S.; Min, S.C. Microbial Decontamination of Onion Powder Using Microwave-Powered Cold Plasma Treatments. Food Microbiol. 2017, 62, 112-123. [CrossRef]

124. Yong, H.I.; Lee, H.; Park, S.; Park, J.; Choe, W.; Jung, S.; Jo, C. Flexible Thin-Layer Plasma Inactivation of Bacteria and Mold Survival in Beef Jerky Packaging and Its Effects on the Meat's Physicochemical Properties. Meat Sci. 2017, 123, 151-156. [CrossRef] [PubMed]

125. Sohbatzadeh, F.; Mirzanejhad, S.; Shokri, H.; Nikpour, M. Inactivation of Aspergillus flavus Spores in a Sealed Package by Cold Plasma Streamers. J. Theor. Appl. Phys. 2016, 10, 99-106. [CrossRef]

126. Kim, J.E.; Lee, D.U.; Min, S.C. Microbial Decontamination of Red Pepper Powder by Cold Plasma. Food Microbiol. 2014, 38 , 128-136. [CrossRef] [PubMed]

127. Suhem, K.; Matan, N.; Nisoa, M.; Matan, N. Inhibition of Aspergillus flavus on Agar Media and Brown Rice Cereal Bars Using Cold Atmospheric Plasma Treatment. Int. J. Food Microbiol. 2013, 161, 107-111. [CrossRef] [PubMed]

128. Hosseini, S.I.; Farrokhi, N.; Shokri, K.; Khani, M.R.; Shokri, B. Cold Low Pressure $\mathrm{O}_{2}$ Plasma Treatment of Crocus sativus: An Efficient Way to Eliminate Toxicogenic Fungi with Minor Effect on Molecular and Cellular Properties of Saffron. Food Chem. 2018, 257, 310-315. [CrossRef] [PubMed]

129. Starek, A.; Sagan, A.; Andrejko, D.; Chudzik, B.; Kobus, Z.; Kwiatkowski, M.; Terebun, P.; Pawłat, J. Possibility to Extend the Shelf Life of NFC Tomato Juice Using Cold Atmospheric Pressure Plasma. Sci. Rep. 2020, 10, 20959. [CrossRef]

130. Park, S.Y.; Ha, S.-D. Application of Cold Oxygen Plasma for the Reduction of Cladosporium cladosporioides and Penicillium citrinum on the Surface of Dried Filefish (Stephanolepis cirrhifer) Fillets. Int. J. Food Sci. Technol. 2015, 50, 966-973. [CrossRef]

131. Choi, E.J.; Park, H.W.; Kim, S.B.; Ryu, S.; Lim, J.; Hong, E.J.; Byeon, Y.S.; Chun, H.H. Sequential Application of Plasma-Activated Water and Mild Heating Improves Microbiological Quality of Ready-to-Use Shredded Salted Kimchi Cabbage (Brassica pekinensis L.). Food Control 2019, 98, 501-509. [CrossRef]

132. Siciliano, I.; Spadaro, D.; Prelle, A.; Vallauri, D.; Cavallero, M.C.; Garibaldi, A.; Gullino, M.L. Use of Cold Atmospheric Plasma to Detoxify Hazelnuts from Aflatoxins. Toxins 2016, 8, 125. [CrossRef]

133. Sen, Y.; Onal-Ulusoy, B.; Mutlu, M. Detoxification of Hazelnuts by Different Cold Plasmas and Gamma Irradiation Treatments. Innov. Food Sci. Emerg. Technol. 2019, 54, 252-259. [CrossRef]

134. Puligundla, P.; Lee, T.; Mok, C. Effect of Corona Discharge Plasma Jet Treatment on the Degradation of Aflatoxin B1 on Glass Slides and in Spiked Food Commodities. LWT 2020, 124, 108333. [CrossRef]

135. Hojnik, N.; Modic, M.; Žigon, D.; Kovač, J.; Jurov, A.; Dickenson, A.; Walsh, J.L.; Cvelbar, U. Cold Atmospheric Pressure Plasma-Assisted Removal of Aflatoxin B1 from Contaminated Corn Kernels. Plasma Process. Polym. 2021, 18, 2000163. [CrossRef]

136. Makari, M.; Hojjati, M.; Shahbazi, S.; Askari, H. Elimination of Aspergillus flavus from Pistachio Nuts with Dielectric Barrier Discharge (DBD) Cold Plasma and Its Impacts on Biochemical Indices. J. Food Qual. 2021, 2021, 9968711. [CrossRef]

137. Ten Bosch, L.; Pfohl, K.; Avramidis, G.; Wieneke, S.; Viöl, W.; Karlovsky, P. Plasma-Based Degradation of Mycotoxins Produced by Fusarium, Aspergillus and Alternaria species. Toxins 2017, 9, 97. [CrossRef] [PubMed]

138. Bulson, J.M.; Liveris, D.; Derkatch, I.; Friedman, G.; Geliebter, J.; Park, S.; Singh, S.; Zemel, M.; Tiwari, R.K. Non-Thermal Atmospheric Plasma Treatment of Onychomycosis in an In Vitro Human Nail Model. Mycoses 2020, 63, 225-232. [CrossRef] [PubMed] 
139. Lux, J.; Dobiáš, R.; Kuklová, I.; Litvik, R.; Scholtz, V.; Soušková, H.; Khun, J.; Mrázek, J.; Kantorová, M.; Jaworská, P.; et al. Inactivation of Dermatophytes Causing Onychomycosis and Its Therapy Using Non-Thermal Plasma. J. Fungi 2020, 6, 214. [CrossRef] [PubMed]

140. Xiong, Z.; Roe, J.; Grammer, T.C.; Graves, D.B. Plasma Treatment of Onychomycosis. Plasma Process. Polym. 2016, $13,588-597$. [CrossRef]

141. Scholtz, V.; Soušková, H.; Hubka, V.; Švarcová, M.; Julák, J. Inactivation of Human Pathogenic Dermatophytes by Non-Thermal Plasma. J. Microbiol. Methods 2015, 119, 53-58. [CrossRef]

142. Sun, P.; Sun, Y.; Wu, H.; Zhu, W.; Lopez, J.L.; Liu, W.; Zhang, J.; Li, R.; Fang, J. Atmospheric Pressure Cold Plasma as an Antifungal Therapy. Appl. Phys. Lett. 2011, 98, 021501. [CrossRef]

143. Ouf, S.A.; El-Adly, A.A.; Mohamed, A.H. Inhibitory Effect of Silver Nanoparticles Mediated by Atmospheric Pressure Air Cold Plasma Jet Against Dermatophyte Fungi. J. Med. Microbiol. 2015, 64, 1151-1161. [CrossRef]

144. Scholtz, V.; Soušková, H.; Švarcová, M.; Kříha, V.; Živná, H.; Julák, J. Inactivation of Dermatophyte Infection by Nonthermal Plasma on Animal Model. Med. Mycol. 2017, 55, 422-428. [CrossRef]

145. Borges, A.C.; Lima, G.M.G.; Nishime, T.M.C.; Gontijo, A.V.L.; Kostov, K.G.; Koga-Ito, C.Y. Amplitude-Modulated Cold Atmospheric Pressure Plasma Jet for Treatment of Oral Candidiasis: In Vivo Study. PLoS ONE 2018, 13, e0199832. [CrossRef]

146. Daeschlein, G.; Scholz, S.; von Woedtke, T.; Niggemeier, M.; Kindel, E.; Brandenburg, R.; Weltmann, K.D.; Junger, M. In Vitro Killing of Clinical Fungal Strains by Low-Temperature Atmospheric-Pressure Plasma Jet. IEEE Trans. Plasma Sci. 2011, 39, 815-821. [CrossRef]

147. Lee, O.J.; Ju, H.W.; Khang, G.; Sun, P.P.; Rivera, J.; Cho, J.H.; Park, S.J.; Eden, J.G.; Park, C.H. An Experimental Burn WoundHealing Study of Non-Thermal Atmospheric Pressure Microplasma Jet Arrays. J. Tissue Eng. Regen. Med. 2016, 10, 348-357. [CrossRef] [PubMed]

148. Ali, A.; Hong, Y.; Park, J.; Lee, S.; Choi, E. A Novel Approach to Inactivate the Clinical Isolates of Trichophyton mentagrophytes and Trichophyton rubrum by Using Non-Thermal Plasma. J. Microb. Biochem. Technol. 2014, 6, 314-319.

149. Han, L.; Patil, S.; Keener, K.M.; Cullen, P.J.; Bourke, P. Bacterial Inactivation by High-Voltage Atmospheric Cold Plasma: Influence of Process Parameters and Effects on Cell Leakage and DNA. J. Appl. Microbiol. 2014, 116, 784-794. [CrossRef]

150. Boch, T.; Tennert, C.; Vach, K.; Al-Ahmad, A.; Hellwig, E.; Polydorou, O. Effect of Gaseous Ozone on Enterococcus faecalis Biofilm-an In Vitro Study. Clin. Oral Investig. 2016, 20, 1733-1739. [CrossRef]

151. Thompson, C.M.; Tischler, A.H.; Tarnowski, D.A.; Mandel, M.J.; Visick, K.L. Nitric Oxide Inhibits Biofilm Formation by Vibrio fischeri via the Nitric Oxide Sensor HnoX. Mol. Microbiol. 2019, 111, 187-203. [CrossRef]

152. Mravlje, J.; Regvar, M.; Starič, P.; Mozetič, M.; Vogel-Mikuš, K. Cold Plasma Affects Germination and Fungal Community Structure of Buckwheat Seeds. Plants 2021, 10, 851. [CrossRef] [PubMed]

153. Hojnik, N.; Modic, M.; Tavčar-Kalcher, G.; Babič, J.; Walsh, J.L.; Cvelbar, U. Mycotoxin Decontamination Efficacy of Atmospheric Pressure Air Plasma. Toxins 2019, 11, 219. [CrossRef]

154. Moisan, M.; Barbeau, J.; Moreau, S.; Pelletier, J.; Tabrizian, M.; Yahia, L.H. Low-Temperature Sterilization Using Gas Plasmas: A Review of the Experiments and an Analysis of the Inactivation Mechanisms. Int. J. Pharm. 2001, 226, 1-21. [CrossRef]

155. Hertwig, C.; Meneses, N.; Mathys, A. Cold Atmospheric Pressure Plasma and Low Energy Electron Beam as Alternative Nonthermal Decontamination Technologies for Dry Food Surfaces: A Review. Trends Food Sci. Technol. 2018, 77, 131-142. [CrossRef]

156. Liao, X.; Liu, D.; Xiang, Q.; Ahn, J.; Chen, S.; Ye, X.; Ding, T. Inactivation Mechanisms of Non-Thermal Plasma on Microbes: A Review. Food Control 2017, 75, 83-91. [CrossRef]

157. Scholtz, V.; Šerá, B.; Khun, J.; Šerý, M.; Julák, J. Effects of Nonthermal Plasma on Wheat Grains and Products. J. Food Qual. 2019, 2019, 7917825. [CrossRef]

158. Gow, N.A.R.; Netea, M.G. Medical Mycology and Fungal Immunology: New Research Perspectives Addressing a Major World Health Challenge. Philos. Trans. R. Soc. Lond. B Biol. Sci. 2016, 371, 20150462. [CrossRef] [PubMed]

159. Köhler, J.R.; Casadevall, A.; Perfect, J. The Spectrum of Fungi That Infects Humans. Cold Spring Harb. Perspect. Med. 2014, 5, a019273. [CrossRef] [PubMed]

160. Liu, C.-M.; Nishida, Y.; Iwasaki, K.; Sung, W.; Wu, F.-P. Characteristics of DC or Pulsed-Type High-Electric Field Plasma and Its Application to Air Cleaning System. IEEE Trans. Plasma Sci. 2018, 47, 1121-1128. [CrossRef]

161. Bernhardt, T.; Semmler, M.L.; Schäfer, M.; Bekeschus, S.; Emmert, S.; Boeckmann, L. Plasma Medicine: Applications of Cold Atmospheric Pressure Plasma in Dermatology. Oxid. Med. Cell. Longev. 2019, 2019, 3873928. [CrossRef]

162. Borges, A.C.; Kostov, K.G.; Pessoa, R.S.; De Abreu, G.M.A.; Lima, G.M.G.; Figueira, L.W.; Koga-Ito, C.Y. Applications of Cold Atmospheric Pressure Plasma in Dentistry. Appl. Sci. 2021, 11, 1975. [CrossRef]

163. Heinlin, J.; Isbary, G.; Stolz, W.; Morfill, G.; Landthaler, M.; Shimizu, T.; Steffes, B.; Nosenko, T.; Zimmermann, J.L.; Karrer, S. Plasma Applications in Medicine with a Special Focus on Dermatology. J. Eur. Acad. Dermatol. Venereol. 2011, 25, 1-11. [CrossRef]

164. von Woedtke, T.; Emmert, S.; Metelmann, H.; Rupf, S.; Weltmann, K. Perspectives on Cold Atmospheric Plasma (CAP) Applications in Medicine. Phys. Plasmas 2020, 27, 070601. [CrossRef]

165. Ranieri, P.; Sponsel, N.; Kizer, J.; Rojas-Pierce, M.; Hernández, R.; Gatiboni, L.; Grunden, A.; Stapelmann, K. Plasma Agriculture: Review from the Perspective of the Plant and Its Ecosystem. Plasma Process. Polym. 2021, 18, 2000162. [CrossRef] 
166. Koskey, G.; Mburu, S.W.; Awino, R.; Njeru, E.M.; Maingi, J.M. Potential Use of Beneficial Microorganisms for Soil Amelioration, Phytopathogen Biocontrol, and Sustainable Crop Production in Smallholder Agroecosystems. Front. Sustain. Food Syst. 2021, 5, 130. [CrossRef]

167. Marco, M.L. Defining How Microorganisms Benefit Human Health. Microb. Biotechnol. 2021, 14, 35-40. [CrossRef]

168. Veerana, M.; Lim, J.S.; Choi, E.H.; Park, G. Aspergillus oryzae Spore Germination Is Enhanced by Non-Thermal Atmospheric Pressure Plasma. Sci. Rep. 2019, 9, 11184. [CrossRef] [PubMed]

169. Veerana, M.; Choi, E.H.; Park, G. Influence of Non-Thermal Atmospheric Pressure Plasma Jet on Extracellular Activity of $\alpha$-Amylase in Aspergillus oryzae. Appl. Sci. 2021, 11, 691. [CrossRef]

170. Hu, Z.C.; Li, W.J.; Zou, S.P.; Niu, K.; Zheng, Y.G. Mutagenesis of Echinocandin B Overproducing Aspergillus nidulans Capable of Using Starch as Main Carbon Source. Prep. Biochem. Biotechnol. 2020, 50, 745-752. [CrossRef]

171. Shi, F.; Tan, J.; Chu, J.; Wang, Y.; Zhuang, Y.; Zhang, S. A Qualitative and Quantitative High-Throughput Assay for Screening of Gluconate High-Yield Strains by Aspergillus niger. J. Microbiol. Methods 2015, 109, 134-139. [CrossRef]

172. Zhu, X.; Arman, B.; Chu, J.; Wang, Y.; Zhuang, Y. Development of a Method for Efficient Cost-Effective Screening of Aspergillus niger Mutants Having Increased Production of Glucoamylase. Biotechnol. Lett. 2017, 39, 739-744. [CrossRef]

173. Feng, W.; Liang, J.; Wang, B.; Chen, J. Improvement of Kojic Acid Production in Aspergillus oryzae AR-47 Mutant Strain by Combined Mutagenesis. Bioprocess Biosyst. Eng. 2019, 42, 753-761. [CrossRef]

174. Shu, L.; Si, X.; Yang, X.; Ma, W.; Sun, J.; Zhang, J.; Xue, X.; Wang, D.; Gao, Q. Enhancement of Acid Protease Activity of Aspergillus oryzae Using Atmospheric and Room Temperature Plasma. Front. Microbiol. 2020, 11, 1418. [CrossRef]

175. Li, X.; Zheng, K.; Lai, C.; Ouyang, J.; Yong, Q. Improved Itaconic Acid Production from Undetoxified Enzymatic Hydrolysate of Steam-Exploded Corn Stover Using an Aspergillus terreus Mutant Generated by Atmospheric and Room Temperature Plasma. BioResources 2016, 11, 9047-9058. [CrossRef]

176. Li, H.; Li, T.; Zuo, H.; Xiao, S.; Guo, M.; Jiang, M.; Li, Z.; Li, Y.; Zou, X. A Novel Rhodamine-Based Fluorescent pH Probe for High-Throughput Screening of High-Yield Polymalic Acid Strains from Random Mutant Libraries. RSC Adv. 2016, 6, 94756-94762. [CrossRef]

177. Qiang, W.; Ling-ran, F.; Luo, W.; Han-guang, L.; Lin, W.; Ya, Z.; Xiao-Bin, Y. Mutation Breeding of Lycopene-Producing Strain Blakeslea trispora by a Novel Atmospheric and Room Temperature Plasma (ARTP). Appl. Biochem. Biotechnol. 2014, 174, 452-460. [CrossRef]

178. Luo, Z.; Zeng, W.; Du, G.; Liu, S.; Fang, F.; Zhou, J.; Chen, J. A High-Throughput Screening Procedure for Enhancing Pyruvate Production in Candida Glabrata by Random Mutagenesis. Bioprocess Biosyst. Eng. 2017, 40, 693-701. [CrossRef] [PubMed]

179. Luo, Z.; Liu, S.; Du, G.; Zhou, J.; Chen, J. Identification of a Polysaccharide Produced by the Pyruvate Overproducer Candida Glabrata CCTCC M202019. Appl. Microbiol. Biotechnol. 2017, 101, 4447-4458. [CrossRef]

180. Zheng, S.; Jiang, B.; Zhang, T.; Chen, J. Combined Mutagenesis and Metabolic Regulation to Enhance d-Arabitol Production from Candida parapsilosis. J. Ind. Microbiol. Biotechnol. 2020, 47, 425-435. [CrossRef] [PubMed]

181. Zhang, C.; Qin, J.; Dai, Y.; Mu, W.; Zhang, T. Atmospheric and Room Temperature Plasma (ARTP) Mutagenesis Enables Xylitol Over-Production with Yeast Candida tropicalis. J. Biotechnol. 2019, 296, 7-13. [CrossRef]

182. Huang, W.W.; Ge, X.Y.; Huang, Y.; Chai, X.T.; Zhang, L.; Zhang, Y.X.; Deng, L.N.; Liu, C.Q.; Xu, H.; Gao, J. High-Yield Strain of Fusidic Acid Obtained by Atmospheric and Room Temperature Plasma Mutagenesis and the Transcriptional Changes Involved in Improving Its Production in Fungus Fusidium coccineum. J. Appl. Microbiol. 2021, 130, 405-415. [CrossRef]

183. Ma, Y.; Zhang, Q.; Zhang, Q.; He, H.; Chen, Z.; Zhao, Y.; Wei, D.; Kong, M.; Huang, Q. Improved Production of Polysaccharides in Ganoderma lingzhi Mycelia by Plasma Mutagenesis and Rapid Screening of Mutated Strains Through Infrared Spectroscopy. PLoS ONE 2018, 13, e0204266. [CrossRef] [PubMed]

184. Qin, T.; Song, P.; Wang, X.; Ji, X.; Ren, L.; Huang, H. Protoplast Mutant Selection of Glarea lozoyensis and Statistical Optimization of Medium for Pneumocandin B0 Yield-Up. Biosci. Biotechnol. Biochem. 2016, 80, 2241-2246. [CrossRef] [PubMed]

185. Zhu, L.; Wu, D.; Zhang, H.; Li, Q.; Zhang, Z.; Liu, Y.; Zhou, S.; Wang, W.; Li, Z.; Yang, Y. Effects of Atmospheric and Room Temperature Plasma (ARTP) Mutagenesis on Physicochemical Characteristics and Immune Activity In Vitro of Hericium erinaceus Polysaccharides. Molecules 2019, 24, 262. [CrossRef] [PubMed]

186. Li, X.; Liu, R.; Li, J.; Chang, M.; Liu, Y.; Jin, Q.; Wang, X. Enhanced Arachidonic Acid Production from Mortierella alpina Combining Atmospheric and Room Temperature Plasma (ARTP) and Diethyl Sulfate Treatments. Bioresour. Technol. 2015, 177, 134-140. [CrossRef]

187. Gu, L.S.; Tan, M.Z.; Li, S.H.; Zhang, T.; Zhang, Q.Q.; Li, C.X.; Luo, X.M.; Feng, J.X.; Zhao, S. ARTP/EMS-Combined Multiple Mutagenesis Efficiently Improved Production of Raw Starch-Degrading Enzymes in Penicillium oxalicum and Characterization of the Enzyme-Hyperproducing Mutant. Biotechnol. Biofuels 2020, 13, 187. [CrossRef] [PubMed]

188. Zhang, G.; Lin, Y.; Qi, X.; Wang, L.; He, P.; Wang, Q.; Ma, Y. Genome Shuffling of the Nonconventional Yeast Pichia anomala for Improved Sugar Alcohol Production. Microb. Cell Fact. 2015, 14, 112. [CrossRef] [PubMed]

189. Qi, F.; Kitahara, Y.; Wang, Z.; Zhao, X.; Du, W.; Liu, D. Novel Mutant Strains of Rhodosporidium toruloides by Plasma Mutagenesis Approach and Their Tolerance for Inhibitors in Lignocellulosic Hydrolyzate. J. Chem. Technol. Biotechnol. 2014, 89, 735-742. [CrossRef]

190. Kitahara, Y.; Yin, T.; Zhao, X.; Wachi, M.; Du, W.; Liu, D. Isolation of Oleaginous Yeast (Rhodosporidium toruloides) Mutants Tolerant of Sugarcane Bagasse Hydrolysate. Biosci. Biotechnol. Biochem. 2014, 78, 336-342. [CrossRef] [PubMed] 
191. Qi, F.; Zhao, X.; Kitahara, Y.; Li, T.; Ou, X.; Du, W.; Liu, D.; Huang, J. Integrative Transcriptomic and Proteomic Analysis of the Mutant Lignocellulosic Hydrolyzate-Tolerant Rhodosporidium toruloides. Eng. Life Sci. 2017, 17, 249-261. [CrossRef] [PubMed]

192. Zhang, C.; Shen, H.; Zhang, X.; Yu, X.; Wang, H.; Xiao, S.; Wang, J.; Zhao, Z.K. Combined Mutagenesis of Rhodosporidium toruloides for Improved Production of Carotenoids and Lipids. Biotechnol. Lett. 2016, 38, 1733-1738. [CrossRef] [PubMed]

193. Wang, Q.; Liu, D.; Yang, Q.; Wang, P. Enhancing Carotenoid Production in Rhodotorula mucilaginosa KC8 by Combining Mutation and Metabolic Engineering. Ann. Microbiol. 2017, 67, 425-431. [CrossRef]

194. Liang, M.H.; Liang, Y.J.; Chai, J.Y.; Zhou, S.S.; Jiang, J.G. Reduction of Methanol in Brewed Wine by the Use of Atmospheric and Room-Temperature Plasma Method and the Combination Optimization of Malt with Different Adjuncts. J. Food Sci. 2014, 79, M2308-M2314. [CrossRef] [PubMed]

195. Xu, W.; Jia, H.; Zhang, L.; Wang, H.; Tang, H.; Zhang, L. Effects of GSH1 and GSH2 Gene Mutation on Glutathione Synthetases Activity of Saccharomyces cerevisiae. Protein J. 2017, 36, 270-277. [CrossRef]

196. Li, T.; Chen, L.; Wu, D.; Dong, G.; Chen, W.; Zhang, H.; Yang, Y.; Wu, W. The Structural Characteristics and Biological Activities of Intracellular Polysaccharide Derived from Mutagenic Sanghuangporous sanghuang Strain. Molecules 2020, 25, 3693. [CrossRef]

197. Ma, X.J.; Zhang, H.M.; Lu, X.F.; Han, J.; Zhu, H.X.; Wang, H.; Yao, R.S. Mutant Breeding of Starmerella bombicola by Atmospheric and Room-Temperature Plasma (ARTP) for Improved Production of Specific or Total Sophorolipids. Bioprocess Biosyst. Eng. 2020, 43, 1869-1883. [CrossRef]

198. Zou, Z.; Zhao, Y.; Zhang, T.; Xu, J.; He, A.; Deng, Y. Efficient Isolation and Characterization of a Cellulase Hyperproducing Mutant Strain of Trichoderma reesei. J. Microbiol. Biotechnol. 2018, 28, 1473-1481. [CrossRef]

199. Xu, F.; Wang, J.; Chen, S.; Qin, W.; Yu, Z.; Zhao, H.; Xing, X.; Li, H. Strain Improvement for Enhanced Production of Cellulase in Trichoderma viride. Prikl. Biokhim. Mikrobiol. 2011, 47, 61-65. [CrossRef] [PubMed]

200. Xu, F.; Jin, H.; Li, H.; Tao, L.; Wang, J.; Lv, J.; Chen, S. Genome Shuffling of Trichoderma viride for Enhanced Cellulase Production. Ann. Microbiol. 2012, 62, 509-515. [CrossRef]

201. Zeng, W.; Du, G.; Chen, J.; Li, J.; Zhou, J. A High-Throughput Screening Procedure for Enhancing $\alpha$-Ketoglutaric Acid Production in Yarrowia lipolytica by Random Mutagenesis. Process Biochem. 2015, 50, 1516-1522. [CrossRef]

202. Zeng, W.; Fang, F.; Liu, S.; Du, G.; Chen, J.; Zhou, J. Comparative Genomics Analysis of a Series of Yarrowia lipolytica WSH-Z06 Mutants with Varied Capacity for $\alpha$-Ketoglutarate Production. J. Biotechnol. 2016, 239, 76-82. [CrossRef] [PubMed]

203. Liu, X.; Lv, J.; Xu, J.; Xia, J.; Dai, B.; Xu, X.; Xu, J. Erythritol Production by Yarrowia lipolytica Mutant strain M53 Generated Through Atmospheric and Room Temperature Plasma Mutagenesis. Food Sci. Biotechnol. 2017, 26, 979-986. [CrossRef] [PubMed]

204. Arnau, J.; Yaver, D.; Hjort, C.M. Strategies and Challenges for the Development of Industrial Enzymes Using Fungal Cell Factories. In Grand Challenges in Fungal Biotechnology; Nevalainen, H., Ed.; Springer: Cham, Switzerland, 2019; pp. 179-210. [CrossRef]

205. Zhang, X.; Zhang, X.F.; Li, H.P.; Wang, L.Y.; Zhang, C.; Xing, X.H.; Bao, C.Y. Atmospheric and Room Temperature Plasma (ARTP) as a New Powerful Mutagenesis Tool. Appl. Microbiol. Biotechnol. 2014, 98, 5387-5396. [CrossRef]

206. Wende, K.; Bekeschus, S.; Schmidt, A.; Jatsch, L.; Hasse, S.; Weltmann, K.D.; Masur, K.; von Woedtke, T. Risk Assessment of a Cold Argon Plasma Jet in Respect to Its Mutagenicity. Mutat. Res. Genet. Toxicol. Environ. Mutagen. 2016, 798, 48-54. [CrossRef]

207. Bruggeman, P.J.; Kushner, M.J.; Locke, B.R.; Gardeniers, J.G.E.; Graham, W.G.; Graves, D.B.; Hofman-Caris, R.C.H.M.; Maric, D.; Reid, J.P.; Ceriani, E.; et al. Plasma-Liquid Interactions: A Review and Roadmap. Plasma Sources Sci. Technol. 2016, $25,053002$. [CrossRef]

208. Kondeti, V.S.S.K.; Phan, C.Q.; Wende, K.; Jablonowski, H.; Gangal, U.; Granick, J.L.; Hunter, R.C.; Bruggeman, P.J. Long-Lived and Short-Lived Reactive Species Produced by a Cold Atmospheric Pressure Plasma Jet for the Inactivation of Pseudomonas aeruginosa and Staphylococcus aureus. Free Radic. Biol. Med. 2018, 124, 275-287. [CrossRef] [PubMed]

209. Gaunt, L.F.; Beggs, C.B.; Georghiou, G.E. Bactericidal Action of the Reactive Species Produced by Gas-Discharge Nonthermal Plasma at Atmospheric Pressure: A Review. IEEE Trans. Plasma Sci. 2006, 34, 1257-1269. [CrossRef]

210. Graves, D.B. The Emerging Role of Reactive Oxygen and Nitrogen Species in Redox Biology and Some Implications for Plasma Applications to Medicine and Biology. J. Phys. D Appl. Phys. 2012, 45, 263001. [CrossRef]

211. Xu, H.; Zhu, Y.; Du, M.; Wang, Y.; Ju, S.; Ma, R.; Jiao, Z. Subcellular Mechanism of Microbial Inactivation During Water Disinfection by Cold Atmospheric-Pressure Plasma. Water Res. 2021, 188, 116513. [CrossRef] [PubMed]

212. Dobrynin, D.; Fridman, G.; Friedman, G.; Fridman, A. Physical and Biological Mechanisms of Direct Plasma Interaction with Living Tissue. New J. Phys. 2009, 11, 115020. [CrossRef]

213. Kobzev, E.N.; Kireev, G.V.; Rakitskii, Y.A.; Martovetskaya, I.I.; Chugunov, V.A.; Kholodenko, V.P.; Khramov, M.V.; Akishev, Y.S.; Trushkin, N.I.; Grushin, M.E. Effect of Cold Plasma on the E. coli Cell Wall and Plasma Membrane. Appl. Biochem. Microbiol. 2013, 49, 144-149. [CrossRef] 